\title{
Ricci almost solitons
}

\author{
Stefano Pigola, Marco Rigoli, Michele Rimoldi \\ AND Alberto G. SETti
}

\begin{abstract}
We introduce a natural extension of the concept of gradient Ricci soliton: the Ricci almost soliton. We provide existence and rigidity results, we deduce a-priori curvature estimates and isolation phenomena, and we investigate some topological properties. A number of differential identities involving the relevant geometric quantities are derived. Some basic tools from the weighted manifold theory such as general weighted volume comparisons and maximum principles at infinity for diffusion operators are discussed.
\end{abstract}

Mathematics Subject Classification (2010): 53C21.

\section{Introduction}

Let $(M,\langle\rangle$,$) be a Riemannian manifold. A Ricci soliton structure on M$ is the choice of a smooth vector field $X$ (if any) satisfying the soliton equation

$$
\operatorname{Ric}+\frac{1}{2} L_{X}\langle,\rangle=\lambda\langle,\rangle
$$

for some constant $\lambda \in \mathbb{R}$. Here, Ric denotes the Ricci tensor of $M$ and $L_{X}$ stands for the Lie derivative in the direction of $X$.

In the special case where $X=\nabla f$ for some smooth function $f: M \rightarrow \mathbb{R}$, we say that $(M,\langle\rangle,, \nabla f)$ is a gradient Ricci soliton with potential $f$. In this case the soliton equation (1.1) reads

$$
\operatorname{Ric}+\operatorname{Hess}(f)=\lambda\langle,\rangle \text {. }
$$

Clearly, equations (1.1) and (1.2) can be considered as perturbations of the Einstein equation

$$
\text { Ric }=\lambda\langle,\rangle
$$

and reduce to this latter in case $X$ or $\nabla f$ are Killing vector fields. When $X=0$ or $f$ is constant we call the underlying Einstein manifold a trivial Ricci soliton.

Received March 15, 2010; accepted in revised form July 11, 2011. 
As in the case of Einstein manifolds, Ricci solitons exhibit a certain rigidity. This is expressed by triviality and classification results, or by curvature estimates.

For instance, in the compact case it is well known that an expanding (or steady) Ricci soliton is necessarily trivial (see, e.g., [4]). Generalizations to the complete, non-compact setting can be found in the very recent [27].

On the other hand, since the appearance of the seminal works by R. Hamilton, [11], and G. Perelman, [21], the classification of shrinking gradient Ricci solitons has become the subject of a rapidly increasing investigation. In this direction, we limit ourselves to quote the far-reaching [3] by H.-D. Cao, B.-L. Chen and X.-P. Zhu where a complete classification in the three-dimensional case is given, [33] by Z.-H. Zhang for the extension in the conformally flat, higher dimensional case, and the very recent [16] by O. Munteanu and N. Sesum where, on the base of rigidity works by P. Petersen and W. Wylie, [22,23], and M. Fernández-López and E.GarcíaRío, [7], the authors extend Zhang's classification result to complete shrinkers with harmonic Weyl tensor. The classification of expanding Ricci solitons appears to be more difficult and relatively few results are known. For instance, the reader may consult [22] for the case of constant scalar curvature expanders.

As an instance of curvature estimates, we quote the recent papers by B.-L. Chen, [2], and by Z.-H. Zhang, [34], where it is shown that the scalar curvature of any gradient Ricci soliton is bounded below. In another direction, upper and lower estimates for the infimum of the scalar curvature of a gradient Ricci soliton are obtained in [27], where some triviality and rigidity results at the endpoints are also discussed.

In this paper we propose an extension of the concept of Ricci soliton that, as we are going to explain, appears to be natural and meaningful. First of all we set the following:

Definition 1.1. We say that $(M,\langle\rangle,, \nabla f)$ is a gradient Ricci almost soliton (almost soliton for short) with potential $f$ and soliton function $\lambda$ if (1.2) holds on $M$ with $\lambda$ a smooth function on $M$.

Clearly, the above definition generalizes the notion of gradient Ricci solitons. Note that since a Shur type theorem does not hold, this is a non trivial generalization. One could consider almost Ricci solitons which are not necessarily gradient, replacing the Hessian of $f$ with the Lie derivative $\frac{1}{2} L_{X}\langle$,$\rangle of the metric along a$ vector field, and study the properties of this new object. For instance, it is an interesting problem to find under which conditions an almost Ricci soliton is necessarily gradient. This is going to be the subject of a forthcoming paper. Here we are going to deal with gradient almost Ricci solitons.

We also note that generalizations in different directions have been recently considered. For instance, J. Case, Y.-J. Shu and G. Wei introduce in [1] the concept of a "quasi Einstein manifold", i.e., a Riemannian manifold whose modified BakryEmery Ricci tensor is constant. This definition originates from the study of usual Einstein manifolds that are realized as warped products. Further generalizations have been considered by G. Maschler in [15], where equation (1) is replaced by 
what the author calls the"Ricci-Hessian equation", namely,

$$
\alpha \operatorname{Hess} f+\operatorname{Ric}=\gamma\langle,\rangle
$$

where $\alpha$ and $\gamma$ are functions. Note that since the author is interested in conformal changes of Kähler-Ricci solitons which give rise to new Kähler metrics, the presence of the function $\alpha$ is vital in his investigation.

Extending to our new setting the soliton terminology, we say that the gradient Ricci almost soliton $(M,\langle\rangle,, \nabla f)$ is shrinking, steady or expanding if respectively $\lambda$ is positive, null or negative on $M$. If $\lambda$ has no definitive sign the gradient Ricci almost soliton will be called indefinite. In case $f$ is constant the almost soliton is called trivial and if $\operatorname{dim} M \geq 3$ the underlying manifold $(M,\langle\rangle$,$) is Einstein$ by Schur Theorem. This also suggests that for an almost soliton an appropriate terminology could be that of an almost Einstein manifold.

In view of the fact that the soliton function $\lambda$ is not necessarily constant, one expects that a certain flexibility on the almost soliton structure is allowed and, consequently, the existence of almost solitons is easier to prove than in the classical situation. This feeling is confirmed in Section 2 below where we shall give a number of different examples of almost solitons, showing in particular that all the previous possibilities (shrinking and expanding) with a non-constant soliton function $\lambda$ can indeed occur. On the other hand, the rigidity result contained in Theorem 2.3 below indicates that almost solitons should reveal a reasonably broad generalization of the fruitful concept of classical soliton. In particular, one obtains that not every complete manifold supports an almost soliton structure; see Example 2.4.

The investigation in this paper is mainly concerned with triviality and pointwise curvature estimates of gradient Ricci almost solitons in case $(M,\langle\rangle$,$) is a$ complete, connected manifold. From now on we let $m=\operatorname{dim} M$; we fix an origin $o \in M$ and we let $r(x)$ denote the distance function from $o . B_{r}$ and $\partial B_{r}$ are respectively the geodesic ball of radius $r$ centered at $o$ and its boundary. Given the potential function $f \in C^{\infty}(M)$ we consider the weighted manifold $\left(M,\langle\rangle,, e^{-f} d\right.$ vol $)$, where $d$ vol is the Riemannian volume element. We set

$$
\operatorname{vol}_{f}\left(B_{r}(p)\right)=\int_{B_{r}(p)} e^{-f} d \mathrm{vol}, \quad \operatorname{vol}_{f}\left(\partial B_{r}(p)\right)=\int_{\partial B_{r}(p)} e^{-f} d \operatorname{vol}_{m-1},
$$

where $d \mathrm{vol}_{m-1}$ stands for the $(m-1)$-dimensional Hausdorff measure. Finally we call $f$-laplacian, $\Delta_{f}$, the diffusion operator defined on $u$ by

$$
\Delta_{f} u=e^{f} \operatorname{div}\left(e^{-f} \nabla u\right)=\Delta u-\langle\nabla f, \nabla u\rangle
$$

which is clearly symmetric on $L^{2}\left(M, e^{-f} d\right.$ vol $)$.

Note that in the terminology of weighted manifolds the LHS of (1.2) is the Bakry-Emery Ricci tensor of $\left(M,\langle\rangle,, e^{-f} d \mathrm{vol}\right)$ that is usually indicated with $\mathrm{Ric}_{f}$. We are now ready to state our first result. 
Theorem 1.2. Let $(M,\langle\rangle,, \nabla f)$ be a complete, expanding gradient Ricci almost soliton with soliton function $\lambda$. Let $\alpha, \sigma, \mu \in \mathbb{R}$ be such that

$$
\begin{gathered}
\alpha>-2 ; 0 \leq \sigma \leq 2 / 3 \\
\min \{0,-\alpha\} \leq \mu \leq\left\{\begin{array}{lll}
1-3 \sigma / 2 & \text { if } \sigma \geq \alpha \\
1-\sigma-\alpha / 2 & \text { if } \sigma<\alpha
\end{array}\right.
\end{gathered}
$$

Assume

$$
\begin{gathered}
\limsup _{r(x) \rightarrow+\infty} \frac{|\nabla f|^{2}}{r(x)^{\sigma}} \begin{cases}=0 & \text { if } 0<\sigma \leq 2 / 3 \\
<+\infty & \text { if } \quad \sigma=0\end{cases} \\
-(m-1) B^{2}\left(1+r(x)^{2}\right)^{\frac{\alpha}{2}} \leq \lambda(x) \leq-(m-1) A^{2}\left(1+r(x)^{2}\right)^{-\frac{\mu}{2}}
\end{gathered}
$$

on $M$ for some constants $B \geq A>0$.

Suppose either $m=2$ or

$$
\langle\nabla f, \nabla \lambda\rangle \leq 0 \text { on } M .
$$

Then the almost soliton is trivial.

Note that (1.3) implies that (1.5) is meaningful.

Corollary 1.3. Let $(M,\langle\rangle,, \nabla f)$ be a complete, expanding gradient Ricci soliton such that

$$
\limsup _{r(x) \rightarrow+\infty} \frac{|\nabla f|^{2}}{r(x)^{\sigma}} \begin{cases}=0 & 0<\sigma \leq \frac{2}{3} \\ <+\infty & \sigma=0 .\end{cases}
$$

Then the soliton is trivial.

The case $\sigma=0$ of Corollary 1.3 has been proved in [27]. The next result extends Theorem 3 in [27] to the case of almost solitons; see also [35]. Note that, contrary to [23], we do not assume that the scalar curvature is either constant or bounded.

Theorem 1.4. Let $(M,\langle\rangle,, \nabla f)$ be a complete gradient Ricci almost soliton with scalar curvature $S$ and soliton function $\lambda$ such that $\Delta \lambda \leq 0$ on M. Set

$$
S_{*}=\inf _{M} S, \quad \lambda_{*}=\inf _{M} \lambda, \quad \lambda^{*}=\sup _{M} \lambda
$$

(i) If the almost soliton is expanding with $-\infty<\lambda_{*} \leq \lambda \leq 0, \lambda \not \equiv 0$, then $m \lambda_{*} \leq$ $S_{*} \leq 0$. Moreover, if $m \geq 3$ and there exists $x_{o}$ such that $S\left(x_{o}\right)=S_{*}=m \lambda_{*}$, then the soliton is trivial and $M$ is Einstein.

(ii) If the almost soliton is a steady soliton then $S_{*}=0$. Morever, if $m \geq 3$ and there exists $x_{o}$ such that $S\left(x_{o}\right)=0$, then $M$ is a cylinder over a totally geodesic hypersurface. 
(iii) If the almost soliton is shrinking with $0 \leq \lambda, \lambda \not \equiv 0$, then $0 \leq S_{*} \leq m \lambda^{*}$. Moreover if $m \geq 3$ and there exists $x_{o}$ such that $S\left(x_{o}\right)=S_{*}=0$ then $M$ is isometric to the standard Euclidean space. Finally if $S_{*}=m \lambda^{*}$ and $\left(M,\langle\rangle,, e^{-f} d \mathrm{vol}\right)$ is $f$-parabolic, then the almost soliton is trivial and $(M,\langle\rangle$,$) is compact Einstein.$ This latter case occurs in particular if

$$
A^{2}(1+r(x))^{-\mu} \leq \lambda(x)
$$

on $M$ for some $A>0,0 \leq \mu \leq 1$.

Note that the case $\mu=0$ contains, of course, the soliton case.

Corollary 1.5. In the assumptions of Theorem 1.4, in cases (i) and (iii), $(M,\langle\rangle$, has non negative scalar curvature.

In the next result we shall assume the validity of a weighted Poincaré-Sobolev inequality on $M$.

Theorem 1.6. Let $(M,\langle\rangle,, \nabla f)$ be a complete gradient Ricci almost soliton with soliton function $\lambda$. For some $0 \leq \alpha<1$ assume on $M$ the validity of

$$
\int_{M}|\nabla \varphi|^{2} e^{-f} \geq S(\alpha)^{-1}\left\{\int_{M}|\varphi|^{\frac{2}{1-\alpha}} e^{-f}\right\}^{1-\alpha}
$$

for all $\varphi \in C_{c}^{\infty}(M)$ and some constant $S(\alpha)>0$. Suppose that

$$
\int_{B_{r}}|\nabla f|^{p} e^{-f}=o\left(r^{2}\right)
$$

as $r \rightarrow+\infty$, for some $p>1$, and that

$$
\left\|\lambda_{+}\right\|_{L^{\frac{1}{\alpha}}\left(M, e^{-f} d \mathrm{vol}\right)}<\frac{4}{S(\alpha)} \frac{p-1}{p^{2}},
$$

with $\lambda_{+}(x)=\max \{0, \lambda(x)\}$. Suppose that either $m=2$ or (1.6) is satisfied. Then the almost soliton is trivial and, when $m \geq 3, M$ is Einstein with non-positive Ricci curvature.

As an immediate consequence we obtain:

Corollary 1.7. Let $(M,\langle,\rangle \nabla f)$ be a complete, expanding, gradient Ricci soliton and assume that (1.8) and (1.9) hold for some $0 \leq \alpha<1$ and $p>1$. Then the soliton is trivial.

Remarks 1.8. (a) Note that, according to the variational characterization of the bottom of the spectrum of the $f$-Laplacian, assumption (1.8) with $\alpha=0$ means

$$
\lambda_{1}^{-\Delta_{f}}\left(M, e^{-f} d \mathrm{vol}\right)>0 .
$$


Thus, in particular, inequality (1.8) with $\alpha=0$ holds if the almost soliton $(M,\langle\rangle,, \nabla f)$ is expanding and satisfies:

$$
\operatorname{Sec}_{\mathrm{rad}} \leq-K \leq 0 \text { and } \frac{\partial f}{\partial r} \leq 0 .
$$

This follows from [29, Theorem 3.4].

(b) Condition (1.8) implies that $\operatorname{vol}_{f}(M)=+\infty$. Indeed, let $\varphi \in C_{c}^{\infty}$ be such that $\varphi=1$ on $B_{R}, \varphi=0$ off $B_{2 R},|\nabla \varphi| \leq \frac{C}{R}$. Then, from (1.8)

$$
\begin{aligned}
\frac{C^{2}}{R^{2}} \int_{B_{2 R} \backslash B_{R}} e^{-f} & \geq \int_{M}|\nabla \varphi|^{2} e^{-f} \\
& \geq S(\alpha)^{-1}\left(\int_{M}|\varphi|^{\frac{2}{1-\alpha} e^{-f}}\right)^{1-\alpha} \\
& \geq S(\alpha)^{-1}\left(\int_{B_{R}} e^{-f}\right)^{1-\alpha}
\end{aligned}
$$

i.e.

$$
\frac{C^{2}}{R^{2}}\left\{\operatorname{vol}_{f}\left(B_{2 R}\right)-\operatorname{vol}_{f}\left(B_{R}\right)\right\} \geq S(\alpha)^{-1} \operatorname{vol}_{f}\left(B_{R}\right)^{1-\alpha}
$$

Denoting by $T=\operatorname{Ric}_{M}-\frac{1}{m} S\langle$,$\rangle the trace free Ricci tensor of (M,\langle\rangle$,$) the next$ result is a gap theorem for the values of

$$
|T|^{*}=\sup _{M}|T|
$$

Theorem 1.9. Let $(M,\langle\rangle,, \nabla f)$ be a complete, conformally flat almost soliton with scalar curvature $S$, trace free Ricci tensor $T$ and soliton function $\lambda$ such that

$$
\langle\operatorname{Hess}(\lambda), T\rangle \geq 0
$$

on $M$. Assume $m=\operatorname{dim} M \geq 3$,

$$
\begin{aligned}
& S^{*}=\sup _{M} S<+\infty, \\
& \lambda_{*}=\inf _{M} \lambda>-\infty .
\end{aligned}
$$

Then either $(M,\langle\rangle$,$) is Einstein and the classification of Theorem 2.3$ below applies or

$$
|T|^{*} \geq \frac{1}{2}\left(\sqrt{m(m-1)} \lambda_{*}-S^{*} \frac{m-2}{\sqrt{m(m-1)}}\right) .
$$


Our results will follow from considering elliptic equations or inequalities for various geometric quantities on almost solitons and rely on analytic techniques. This is the same philosophy used, for instance, in [4,22]. More specifically, we will see that the differential (in)equalities at hand naturally involve the $f$-Laplace operator. Since the almost soliton equation means precisely that the $f$-Bakry-Emery Ricci curvature is proportional to the metric tensor, we are naturally led to introduce a number of weighted manifolds tools whose range of applications goes beyond the investigation of almost solitons. This point of view is in the spirit of [31]. An important instance of these tools is represented by the maximum principles at infinity under both weighted Ricci lower bounds and weighted volume growth conditions. As an example, we shall observe the validity of the following:

Theorem 1.10. Let $\left(M,\langle\rangle,, e^{-f} d \mathrm{vol}\right)$ be a complete weighted manifold whose Bakry-Emery Ricci tensor satisfies

$$
\operatorname{Ric}_{f} \geq-(m-1) G(r(x))
$$

where $G$ is a smooth function on $[0,+\infty)$ satisfying
(i) $G(0)>0$
(ii) $G^{\prime}(t) \geq 0$ on $[0,+\infty)$
(iii) $G(t)^{-\frac{1}{2}} \notin L^{1}(+\infty)$
(iv) $\lim \sup _{t \rightarrow+\infty} \frac{t G\left(t^{\frac{1}{2}}\right)}{G(t)}<+\infty$.

Assume also that

$$
|\nabla f| \leq C G(r)^{1 / 2} .
$$

Then, for every smooth function $u$ such that $\sup _{M} u=u^{*}<+\infty$ there exists a sequence $\left\{x_{n}\right\}$ along which
(i) $u\left(x_{k}\right)>u^{*}-\frac{1}{k}$;
(ii) $\left|\nabla u\left(x_{k}\right)\right|<\frac{1}{k}$;
(iii) $\Delta_{f} u\left(x_{k}\right)<\frac{1}{k}$.

The paper is organized as follows. In Section 1 we provide some examples of almost gradient solitons and we prove the rigidity result contained in Theorem 2.3. Section 2 is devoted to derive the basic elliptic equations that we shall use in the proofs of our results. Some of these equations, for $\lambda=$ const., are well known, see for instance $[4,22]$. Others seem to be new, even in this case (see for instance (3.21) below). In Section 3 we give some improved versions of the Laplacian and volume comparison theorems obtained by Wei and Wylie, [31], and we recall a version of a weak maximum principle, recently proved in [14], appropriate for our present purposes. We also prove a weighted version of a result in [25] from which Theorem 1.10 above immediately follows. The proofs of the main geometric results are contained in Sections 3, 4, 5, 6. We end with Section 7 where we extend to almost solitons some topological results known in the classical case.

ACKNOWLEDGEMENTs. The authors are grateful to Manuel Fernández-López for having sent them the preprints [6] and [7]. 


\section{Examples and rigidity of Einstein almost solitons}

Let $M=I \times{ }_{g} \Sigma$ denote the $g$-warped product of the real interval $I \subseteq \mathbb{R}$ with $0 \in I$, and the Riemannian manifold $\left(\Sigma,(,)_{\Sigma}\right)$ of dimension $\operatorname{dim} \Sigma=m$. Namely, the $(m+1)$-dimensional, smooth product manifold $I \times \Sigma$ is endowed with the metric

$$
\langle,\rangle=d t \otimes d t+g(t)^{2}(,)_{\Sigma},
$$

where $t$ is a global parameter of $I$ and $g: I \rightarrow \mathbb{R}_{0}^{+}$is a smooth function. Using the moving-frame formalism, the geometry of $M$ can be described as follows.

Fix the index convention $1 \leq i, j, k, l, \ldots \leq m$ and $1 \leq \alpha, \beta, \gamma, \ldots \leq m+1$. Let $\left\{e_{j}\right\}$ be a local orthonormal frame of $\Sigma$ with dual frame $\left\{\theta^{j}\right\}$ so that $(,)_{\Sigma}=$ $\sum \theta^{j} \otimes \theta^{j}$. We denote the corresponding connection 1-forms by $\theta_{k}^{j}=-\theta_{j}^{k}$ and the curvature 2-forms by $\Theta_{j}^{i}=-\Theta_{i}^{j}$. Accordingly, the structural equations of $\Sigma$ are

$$
\begin{aligned}
& d \theta^{j}=-\theta_{k}^{j} \wedge \theta^{k} \\
& d \theta_{i}^{j}=-\theta_{k}^{j} \wedge \theta_{i}^{k}+\Theta_{i}^{j} .
\end{aligned}
$$

Furthermore, the curvature forms are related to the (components of) the Riemann tensor by

$$
\Theta_{i}^{j}=\frac{1}{2}{ }^{\Sigma} R_{i k l}^{j} \theta^{k} \wedge \theta^{l} .
$$

Let us introduce the local orthonormal coframe $\left\{\varphi^{\alpha}\right\}$ on $M$ such that

$$
\varphi^{j}=g(t) \theta^{j}, \varphi^{m+1}=d t .
$$

The corresponding connection and curvature forms are denoted, respectively, by $\varphi_{\beta}^{\alpha}=-\varphi_{\alpha}^{\beta}$ and $\Phi_{\beta}^{\alpha}=-\Phi_{\alpha}^{\beta}=\frac{1}{2}{ }^{M} R_{\beta \delta \gamma}^{\alpha} \varphi^{\delta} \wedge \varphi^{\gamma}$. A repeated use of exterior differentiations of $\varphi^{\alpha}$ and $\varphi_{\beta}^{\alpha}$ and of the structure equations of $M$ and $\Sigma$, together with the well known characterization of the Levi Civita connection forms, yield

$$
\begin{aligned}
\varphi_{j}^{k} & =\theta_{j}^{k} \\
\varphi_{m+1}^{k} & =\frac{g^{\prime}}{g} \varphi^{k}=-\varphi_{k}^{m+1},
\end{aligned}
$$

and consequently,

$$
\begin{aligned}
\Phi_{j}^{k} & =-\left(\frac{g^{\prime}}{g}\right)^{2} \varphi^{k} \wedge \varphi^{j}+\Theta_{j}^{k} \\
\Phi_{k}^{m+1} & =\left\{\left(\frac{g^{\prime}}{g}\right)^{2}+\left(\frac{g^{\prime}}{g}\right)^{\prime}\right\} \varphi^{k} \wedge \varphi^{m+1}=\frac{g^{\prime \prime}}{g} \varphi^{k} \wedge \varphi^{m+1}=-\Phi_{m+1}^{k} .
\end{aligned}
$$


Let $\left\{E_{\alpha}\right\}$ denote the dual frame of $\left\{\varphi^{\alpha}\right\}$ so that $E_{j}=g(t)^{-1} e_{j}$. Then,

$$
{ }^{M} \operatorname{Ric}_{\alpha \beta}=\Phi_{\alpha}^{\gamma}\left(E_{\gamma}, E_{\beta}\right) \text {, and }{ }^{\Sigma} \operatorname{Ric}_{k t}=g^{2} \Theta_{k}^{j}\left(E_{j}, E_{t}\right) .
$$

It follows from (2.1) that

$$
\begin{aligned}
& { }^{M} \operatorname{Ric}_{k t}=\left\{-(m-1)\left(\frac{g^{\prime}}{g}\right)^{2}-\frac{g^{\prime \prime}}{g}\right\} \delta_{k t}+\frac{1}{g^{2}}{ }^{\Sigma} \operatorname{Ric}_{k t} \\
& { }^{M} \operatorname{Ric}_{m+1 t}=0 \\
& { }^{M} \mathrm{Ric}_{m+1} m+1=-m \frac{g^{\prime \prime}}{g} .
\end{aligned}
$$

In light of these relations, we have that $M$ is Einstein with ${ }^{M} \mathrm{Ric}=-m c\langle$,$\rangle if and$ only if

$$
{ }^{\Sigma} \operatorname{Ric}_{k t}=\left\{(m-1)\left(\frac{g^{\prime}}{g}\right)^{2}+\frac{g^{\prime \prime}}{g}-m c\right\} g^{2} \delta_{k t},
$$

and

$$
g^{\prime \prime}=c g
$$

Therefore

$$
{ }^{\Sigma} \operatorname{Ric}_{k t}=-(m-1)\left(-g^{\prime 2}+c g^{2}\right) \delta_{k t} .
$$

We explicitly note that the general solution of (2.3) is given by

$$
g(t)=g^{\prime}(0) \mathrm{sn}_{-c}(t)+g(0) \mathrm{cn}_{-c}(t),
$$

where

$$
\operatorname{sn}_{k}(t)= \begin{cases}\frac{1}{\sqrt{-k}} \sinh (\sqrt{-k} t) & \text { if } k<0 \\ t & \text { if } k=0 \\ \frac{1}{\sqrt{k}} \sin (\sqrt{k} t) & \text { if } k>0\end{cases}
$$

and

$$
\mathrm{cn}_{k}(t)=\mathrm{sn}_{k}^{\prime}(t) .
$$

Inserting (2.5) into (2.4) we obtain the following:

Lemma 2.1. Let $\left(\Sigma,(,)_{\Sigma}\right)$ be a Riemannian manifold of dimension $m \geq 3$. Consider the warped product $M=I \times{ }_{g} \Sigma$ where $0 \in I \subseteq \mathbb{R}$ and $g: I \rightarrow \mathbb{R}^{+}$is a smooth function. Then, $M$ is Einstein with

$$
{ }^{M} \text { Ric }=-m c\langle,\rangle, \quad c \in \mathbb{R},
$$

if and only

$$
g(t)=g^{\prime}(0) \mathrm{sn}_{-c}(t)+g(0) \mathrm{cn}_{-c}(t)
$$

and $\Sigma$ is Einstein with

$$
{ }^{\Sigma} \text { Ric }=-(m-1)\left\{-g^{\prime}(0)^{2}+c g(0)^{2}\right\}(,)_{\Sigma} .
$$


Now, consider a smooth function $f: M \rightarrow \mathbb{R}$ of the form $f(t, x)=f(t)$. Its Hessian expresses as

$$
\text { Hess }(f)=f^{\prime} \frac{g^{\prime}}{g} \sum \varphi^{k} \otimes \varphi^{k}+f^{\prime \prime} \varphi^{m+1} \otimes \varphi^{m+1} \text {. }
$$

Thus, in case $M$ is an Einstein manifold with ${ }^{M}$ Ric $=-m c\langle$,$\rangle (hence \Sigma$ is Einstein), the almost Ricci soliton equation on $M$ with respect to the potential $f(x, t)=f(t)$ reads

$$
\left\{\begin{array}{l}
f^{\prime} \frac{g^{\prime}}{g}-m c=\lambda \\
f^{\prime \prime}-m c=\lambda
\end{array}\right.
$$

Integrating this latter we deduce

$$
\left\{\begin{array}{l}
f(t)=a \int_{0}^{t} g(s) d s+b \\
\lambda(t)=a g^{\prime}(t)-m c
\end{array}\right.
$$

for some constants $a, b \in \mathbb{R}$. Summarizing, we have obtained the following examples of Einstein, almost Ricci solitons.

Proposition 2.2. Let $g(t): I \rightarrow \mathbb{R}^{+}$be the smooth function defined in (2.6), $0 \in I \subseteq \mathbb{R}$. Let $\left(\Sigma,(,)_{\Sigma}\right)$ be an m-dimensional Einstein manifold satisfying (2.7). Then, the warped product $M=I \times{ }_{g} \Sigma$ is Einstein with ${ }^{M} \mathrm{Ric}=-m c\langle$,$\rangle and$ it is an almost Ricci soliton with potential $f(t)$ and soliton function $\lambda(t)$ defined in (2.9).

The next rigidity theorem, in the complete case, shows that basically there are no further examples when $(M,\langle\rangle$,$) is Einstein.$

Theorem 2.3. Let $(M,\langle\rangle$,$) be a complete, connected, Einstein manifold of dimen-$ sion $m \geq 4$ and

$$
{ }^{M} \mathrm{Ric}=-(m-1) c\langle,\rangle, \quad c \in \mathbb{R} .
$$

Assume that $M$ is an almost Ricci soliton, namely, for some $\lambda \in C^{\infty}(M)$, there is a solution $f \in C^{\infty}(M)$ of the equation

$$
{ }^{M} \operatorname{Ric}+\operatorname{Hess}(f)=\lambda(x)\langle,\rangle .
$$

(a) If $c=0$, then $\lambda$ must be constant and the following possibilities occur:

(a.1) If $\lambda=0$ then $M$ is isometric to a cylinder $\mathbb{R} \times \Sigma$ over a totally geodesic, Ricci flat hypersurface $\Sigma \subset M$. Furthermore, $f(t, x)=a t+b$, for some constants $a, b \in \mathbb{R}$. 
(a.2) If $\lambda=$ const. $\neq 0$ then $M$ is isometric to $\mathbb{R}^{m}$ and

$$
f(x)=\frac{\lambda}{2}|x|^{2}+\langle b, x\rangle+c,
$$

for some $b \in \mathbb{R}^{m}$ and $c \in \mathbb{R}$.

(b) If $c \neq 0$, then either $\lambda$ is constant and the soliton is trivial, or one of the following cases occurs:

(b.1) $c \in \mathbb{R} \backslash\{0\}$ and $M$ is a space-form of constant curvature -c. Furthermore

$$
\left\{\begin{array}{l}
\lambda(x)=a \mathrm{cn}_{-c}(r(x))-(m-1) c \\
f(x)=c^{-1} a \mathrm{cn}_{-c}(r(x))+b,
\end{array}\right.
$$

for some constants $a, b \in \mathbb{R}$. Here, $r(x)$ denotes the distance from a fixed origin.

(b.2) $c>0$ and $M$ is isometric to the warped product $\mathbb{R} \times_{g} \Sigma$ where

$$
g(t)=\frac{g^{\prime}(0)}{\sqrt{c}} \sinh (\sqrt{c} t)+g(0) \cosh (\sqrt{c} t)>0,
$$

and $\Sigma \subset M$ is an Einstein hypersurface with

$$
{ }^{\Sigma} \text { Ric }=-(m-2)\left(-g^{\prime}(0)^{2}+c g(o)^{2}\right) .
$$

Furthermore

$$
\left\{\begin{array}{l}
\lambda(t, x)=a g^{\prime}(t)-m c \\
f(t, x)=a \int_{0}^{t} g(s) d s+b,
\end{array}\right.
$$

for some constants $a, b \in \mathbb{R}$. Here, $t$ is a global coordinate on $\mathbb{R}$.

Proof. By assumption, with respect to a local orthonormal coframe, we have

$$
f_{i j}=((m-1) c+\lambda) \delta_{i j} .
$$

Differentiating both sides and using the commutation rule

$$
f_{i j k}-f_{i k j}=R_{l i j k} f_{l},
$$

we deduce

$$
R_{l i j k} f_{l}=\lambda_{k} \delta_{i j}-\lambda_{j} \delta_{i k} .
$$

Tracing this latter with respect to $i$ and $k$, recalling that $R_{i j}=-(m-1) c \delta_{i j}$, and simplifying we conclude that

$$
c f_{j}=\lambda_{j} .
$$


We now distinguish several cases.

(a) Suppose $c=0$, i.e., $M$ is Ricci flat. Then $\lambda_{j}=0$ proving that $\lambda$ is constant. The soliton equation reads

$$
\operatorname{Hess}(f)=\lambda\langle,\rangle \text {. }
$$

(a.1) In case $\lambda=0$, then $f$ is affine. In particular $|\nabla f|$ is constant proving that either $f$ is constant, and the soliton is trivial, or $f$ has no critical point at all. Suppose this latter case occurs. Up to rescaling $f$ we can assume that $|\nabla f|=1$, i.e., $f$ is a function of distance type. Then, a Cheeger-Gromoll type argument (see (b.2.ii 1 ) below for details) shows that the flow $\phi$ of the vector field $X=\nabla f$ establishes a Riemannian isometry $\phi: \mathbb{R} \times \Sigma \rightarrow M$, where $\Sigma$ is any of the (totally geodesic) level sets of $f$ and $f$ is a linear function of $t$. Finally, since $M$ is Ricci flat then also $\Sigma$ must be Ricci flat. This proves the first part of statement (a) of the Theorem.

(a.2) Assume $\lambda \neq 0$. Then, it is known that $M$ is isometric to $\mathbb{R}^{m}$ and $f(x)$ takes the form given in (2.10). See [30] and the Appendix in [27] for a straightforward proof. The proof of case (a) is completed.

(b) Suppose $c \neq 0$. By (2.15) we have

$$
f=c^{-1} \lambda+d
$$

for some constant $d \in \mathbb{R}$. Inserting into (2.13) gives

$$
\operatorname{Hess}(\lambda)=c\{(m-1) c+\lambda\}\langle,\rangle \text {. }
$$

If $\lambda(x)=$ const., i.e. $M$ is a classical Ricci soliton, then, in view of (2.16), $f$ must be constant and the soliton is trivial.

Assume then that $\lambda(x)$ is nonconstant. Note that the function

$$
v(x)=(m-1) c+\lambda(x)
$$

is a nontrivial solution of

$$
\operatorname{Hess}(v)=c v\langle,\rangle \text {. }
$$

(b.1.i) If $c<0$, then by the classical Obata theorem, [18], $M$ is isometric to a spaceform of constant curvature $-c>0$ and

$$
v(x)=a \cos (\sqrt{-c} r(x))
$$

for some constant $a \neq 0$. Here, $r(x)$ denotes the distance function from a fixed origin. It follows that the functions $\lambda(x)$ and $f(x)$ take the form given in (b.1), (2.11), for $c<0$.

It remains to consider the case $c>0$. Two possibilities can occur: 
(b.1.ii) The function $v$, which is a nontrivial solution of (2.18), $v$ has at least one critical point $o \in M$ and, therefore, it is a nontrivial solution of the problem

$$
\left\{\begin{array}{l}
\operatorname{Hess}(v)=c v\langle,\rangle \\
|\nabla v|(o)=0
\end{array}\right.
$$

with $c>0$. Thus, for every unit speed geodesic $\gamma$ issuing from $o$, the function $y=v \circ \gamma$ satisfies the initial value problem

$$
\left\{\begin{array}{l}
y^{\prime \prime}=c y \\
y(0)=v(o), y^{\prime}(0)=\langle\nabla v(o), \dot{\gamma}(0)\rangle,
\end{array}\right.
$$

and since $v$ is nonconstant, we have must have $v(o) \neq 0$. Using Kanai's version of Obata theorem, [13], we conclude that $M$ is isometric to hyperbolic space of constant curvature $-c<0$ and $v(x)=v(o) \cosh (\sqrt{c} r(x))$ where $r(x)$ is the distance function from $o$. Inserting this expression into (2.17) and (2.16) completes the proof of case (b.1)

(b.2) The function $v$ has no critical points. A classification of $M$ under this assumption, and the corresponding form of $v, f, \lambda$, can be deduced from some works by Ishihara and Tashiro, [12], and Tashiro, [30]. However we provide a concise and complete proof for the sake of completeness. Let $\Sigma=\{v(x)=s\}$ be a non-empty, smooth, level hypersurface.

Note that, up to multiplying $v$ by a non-zero constant, we can always assume that either $s=0$ or $s=1$. A computation that uses (2.18) shows that the integral curves of the complete vector field $X=\nabla v /|\nabla v|$ are unit speed geodesics orthogonal to $\Sigma$. Moreover, the flow of $X$ gives rise to a smooth map $\phi: \mathbb{R} \times \Sigma \rightarrow M$ which coincides with the normal exponential map $\exp ^{\perp}$ of $\Sigma$. In particular, $\phi$ is surjective. Evaluating (2.18) along the integral curve $\phi(t, x)$ issuing from $x \in \Sigma$ we deduce that $y(t)=v(\phi(t, x))$ satisfies

$$
\left\{\begin{array}{l}
y^{\prime \prime}=c y \\
y(0)=s \in\{0,1\} \\
y^{\prime}(0)=|\nabla v|(x)
\end{array}\right.
$$

and therefore

$$
v(\phi(t, x))=|\nabla v|(x) \mathrm{sn}_{-c}(t)+s \mathrm{cn}_{-c}(t) .
$$

Since

$$
\frac{d v(\phi(t, x))}{d t}=|\nabla v| \circ \phi(t, x)>0
$$

it follows from (2.19) that, necessarily, $c>0$. Moreover, if $s=1$ we have the further restriction $|\nabla v|(x) \geq \sqrt{c}$. The function $v$ is strictly increasing 
along the geodesic curves $\phi_{x}(t)$ issuing from $x \in \Sigma$. Whence, it is easy to conclude that $\phi$ is also injective, hence a diffeomorphism. Since $M \approx$ $\mathbb{R} \times \Sigma$ is connected, also $\Sigma$ must be connected. As a consequence, $|\nabla v|$ is constant on $\Sigma$. Indeed, for any smooth curve $\gamma \subset \Sigma$, we have

$$
\begin{aligned}
\frac{d}{d t}(|\nabla v| \circ \gamma) & =\operatorname{Hess}(v)\left(\frac{\nabla v}{|\nabla v|} \circ \gamma, \dot{\gamma}(t)\right) \\
& =c v(\gamma)\left\langle X_{\gamma}, \dot{\gamma}(t)\right\rangle \\
& =0,
\end{aligned}
$$

because $\dot{\gamma}(t) \in T \Sigma$ and $X_{\gamma}$ is orthogonal to $\Sigma$. Therefore $|\nabla v|(x)=a \geq$ $\sqrt{c}$, for every $x \in \Sigma$. Using this information into (2.19) with $c>0$ gives

$$
v(\phi(t, x))=\alpha(t)
$$

where we have set

$$
\alpha(t)=\frac{a}{\sqrt{c}} \sinh (\sqrt{c} t)+s \cosh (\sqrt{c} t) .
$$

In particular, $\phi$ moves $\Sigma$ onto every other level set of $v$. To conclude, we show that

$$
\phi^{*}\langle,\rangle=d t^{2}+\left(\alpha^{\prime}\right)^{2}(t)\langle,\rangle_{\Sigma_{0}},
$$

where $\langle,\rangle_{\Sigma}=\left(\phi_{0}\right)^{*}\langle$,$\rangle denotes the metric induced by M$ on the smooth hypersurface $\Sigma$. Indeed, by the above reasonings (or applying Gauss Lemma) we have

$$
\phi^{*}\langle,\rangle=d t^{2}+\left(\phi_{t}\right)^{*}\langle,\rangle \text {. }
$$

Furthermore, using (2.18), (2.20) and the definition of the Lie derivative, we see that, on $T \Sigma_{\phi_{t}}=X_{\phi_{t}}^{\perp}$,

$$
\frac{d}{d t}\left(\phi_{t}\right)^{*}\langle,\rangle=\frac{2 \alpha^{\prime \prime}}{\alpha^{\prime}} \phi_{t}^{*}\langle,\rangle
$$

Whence, integrating on $[0, t]$ we conclude the validity of (2.21). Summarizing, we have obtained that, if $v$ has no critical point, then $(M,\langle\rangle$,$) is$ isometric to the warped product manifold

$$
\left(\mathbb{R} \times \Sigma, d t^{2}+\alpha^{\prime}(t)^{2}\langle,\rangle_{\Sigma}\right)
$$

with $\Sigma$ a smooth hypersurface of $M$. By assumption, $M$ is Einstein with constant Ricci curvature $-(m-1) c$, therefore $\Sigma$ is Einstein and the expression of its Ricci curvature follows from Lemma 2.1. 
(b.2.ii) To conclude, assume that $v$ possesses at least one critical point $o \in M$ and, therefore, it is a nontrivial solution of the problem

$$
\left\{\begin{array}{l}
\operatorname{Hess}(v)=c v\langle,\rangle \\
|\nabla v|(o)=0,
\end{array}\right.
$$

with $c>0$. Since $v$ is nonconstant, we have $v(o) \neq 0$. Using Kanai version of Obata theorem, [13], we conclude that $M$ is isometric to the hyperbolic space of constant curvature $-c<0$ and $v(x)=v(o) \cosh (\sqrt{c} r(x))$ where $r(x)$ is the distance function from $o$. Inserting this expression into (2.17) and (2.16) completes the proof of case (b) and, hence, of the theorem.

Example 2.4. Let $M$ be any (possibly trivial) quotient of the Riemannian product of standard spheres $\mathbb{S}^{2} \times \mathbb{S}^{2}$ or a non trivial quotient of $\mathbb{S}^{m}$. Then, $M$ is Einstein, and according to Theorem 2.3, (b.1) $M$ has no nontrivial almost Ricci soliton structure.

A similar conclusion holds for possibly trivial quotients of the Riemannian product of standard hyperbolic spaces $\mathbb{H}^{2} \times \mathbb{H}^{2}$. Clearly it suffices to consider $\mathbb{H}^{2} \times \mathbb{H}^{2}$ itself. Since $\mathbb{H}^{2} \times \mathbb{H}^{2}$ is Einstein with Ric $=-\langle$,$\rangle , if it had the structure$ of a nontrivial almost soliton structure, by Theorem 2.3 it would be isometric to the warped product $\mathbb{R} \times{ }_{g} \Sigma$ where $\Sigma$ is a 3 dimensional Einstein hypersurface and $g$ has the form given in the statement of the Theorem. It follows that $\Sigma$ has constant negative curvature, and, from the expression of the Riemann tensor of a warped product (see e.g., [20]), $\mathbb{R} \times_{g} \Sigma \approx \mathbb{H}^{2} \times \mathbb{H}^{2}$ would have strictly negative sectional curvature, which is clearly impossible. Notice that the above reasoning shows that in case $\mathbf{b . 2}$ if $m=4$ and $M$ is simply connected then $\Sigma$ is a hyperbolic space.

Now, suppose that we are given a warped product $M=I \times{ }_{g} \Sigma$ where $(\Sigma,()$, is an $m$-dimensional Einstein manifold and $0 \in I$. If $m \geq 3$, then, for some constant $a$,

$$
{ }^{\Sigma \mathrm{Ric}}=-(m-1) a(,)_{\Sigma} .
$$

According to Lemma 2.1 in order that $M$ be Einstein with ${ }^{M}$ Ric $=-m c\langle$,$\rangle for$ some $c \in \mathbb{R}, g$ must be given by (2.6) and $c g(0)^{2}-g^{\prime}(0)^{2}=a$. Therefore if (2.6) is not satisfied, then $M$ is not Einstein. We consider a function $f(x, t)=f(t)$, so that, using (2.2) and (2.8) we see that to give $M=I \times{ }_{g} \Sigma$ the structure of an almost soliton we need to solve the system

$$
\left\{\begin{array}{l}
f^{\prime} \frac{g^{\prime}}{g}=\lambda+(m-1)\left(\frac{g^{\prime}}{g}\right)^{2}+\frac{g^{\prime \prime}}{g}+\frac{(m-1) a}{g^{2}} \\
f^{\prime \prime}=\lambda+m \frac{g^{\prime \prime}}{g}
\end{array}\right.
$$

on $I$. Subtracting the first equation from the second we obtain

$$
\left(\frac{f^{\prime}}{g}\right)^{\prime}=(m-1) \frac{g g^{\prime \prime}-\left(g^{\prime}\right)^{2}-a}{g^{3}}=(m-1) h(t)
$$


on I, and integrating

$$
f(t)=B+\int_{0}^{t} g(s)\left[A+(m-1) \int_{0}^{s} \frac{g^{\prime \prime} g-\left(g^{\prime}\right)^{2}-a}{g^{3}} d x\right] d s
$$

for some constants $A, B \in \mathbb{R}$. Going back to (2.22) we then deduce

$$
\lambda(t)=-(m-1) \frac{\left(g^{\prime}\right)^{2}+a}{g^{2}}-\frac{g^{\prime \prime}}{g}+g^{\prime}\left[A+(m-1) \int_{0}^{t} \frac{g^{\prime \prime} g-\left(g^{\prime}\right)^{2}-a}{g^{3}} d x\right] .
$$

Summarizing we have obtained the following new set of examples.

Example 2.5. Let $M=I \times{ }_{g} \Sigma^{m}$ where $\Sigma^{m}$ is an Einstein manifold satisfying ${ }^{\Sigma}$ Ric $=-(m-1) a$. Then, $M$ supports an almost soliton structure $f^{\prime} \frac{\partial}{\partial t}$ with soliton function $\lambda(t)$ where $f(t)$ and $\lambda(t)$ are defined respectively in (2.23) and (2.24).

Remark 2.6. As observed above, if $g$ does not satisfy (2.6), these almost solitons are not Einstein hence necessarily different from those produced in Proposition 2.2 above. We also note that if $\Sigma$ is the standard $(m-1)$-dimensional sphere, and $g$ is defined on $I=[-1,+\infty)$ satisfies $g^{(2 k)}(-1)=0, g^{\prime}(-1)=1$ then we obtain a model manifold in the sense of Greene and $\mathrm{Wu}$ (with radial variable $r=t+1$ ), and the almost soliton structure, which is in general defined only on $(-1,+\infty)$ extends to $[-1,+\infty)$ provided the functions $f$ and $\lambda$ can be smoothly extended in $t=-1$. We note that expanding the function $h$ as $t \rightarrow-1^{+}$we obtain that

$$
h(t) \sim \frac{-a-1+o\left((t-1)^{3}\right)}{(t-1)^{3}} .
$$

Thus $h$ integrable in a neighborhood of $t=-1$ and $f$ and $\lambda$ can be extended to $t=-1$ if and only if $a=-1$.

\section{Some basic formulas}

The aim of this section is to prove some basic formulas for gradient Ricci almost solitons. Some of them are well known for solitons, but we have chosen to reproduce computations here since in our more general setting $\lambda$ is a function and significant extra terms appear along the way. Throughout this section computations are performed with the method of the moving frame in a local orthonormal coframe for the metric $\langle$,$\rangle .$

Lemma 3.1. Let $(M,\langle\rangle,, \nabla f)$ be a gradient Ricci almost soliton. Then

$$
\frac{1}{2} \Delta_{f}|\nabla f|^{2}=|\operatorname{Hess}(f)|^{2}-\lambda|\nabla f|^{2}-(m-2)\langle\nabla \lambda, \nabla f\rangle .
$$


Proof. We recall the defining equations

$$
R_{i j}=\lambda \delta_{i j}-f_{i j}
$$

Taking covariant derivatives

$$
R_{i j, k}=\lambda_{k} \delta_{i j}-f_{i j k} .
$$

Tracing with respect to $j$ and $k$

$$
R_{i k, k}=\lambda_{i}-f_{i k k} .
$$

Next tracing the second Bianchi identities

$$
R_{i j k l, s}+R_{i j l s, k}+R_{i j s k, l}=0
$$

with respect to $i$ and $s$ we have

$$
R_{i j k l, i}=R_{j l, k}-R_{j k, l}
$$

and tracing again with respect to $j$ and $l$

$$
2 R_{i k, i}=S_{k},
$$

where $S$ denotes the scalar curvature. Using the commutation relations

$$
R_{i j, k}=R_{j i, k}
$$

we then deduce

$$
R_{k i, i}=\frac{1}{2} S_{k} .
$$

Using (2.14) and (3.6) into (3.4) we finally obtain

$$
\frac{1}{2} S_{i}=\lambda_{i}-f_{k k i}-f_{t} R_{t i} .
$$

Now, tracing (3.3) with respect to $i$ and $j$ yields

$$
S_{i}=m \lambda_{i}-f_{k k i}
$$

so that, substituting into (3.7) gives

$$
S_{i}=2(m-1) \lambda_{i}+2 f_{k} R_{k i} .
$$

In particular, from (3.9) we obtain

$$
\langle\nabla S, \nabla f\rangle=2(m-1)\langle\nabla \lambda, \nabla f\rangle+2 \operatorname{Ric}(\nabla f, \nabla f) .
$$


Next we recall Bochner formula

$$
\frac{1}{2} \Delta|\nabla f|^{2}=|\operatorname{Hess}(f)|^{2}+\operatorname{Ric}(\nabla f, \nabla f)+\langle\nabla \Delta f, \nabla f\rangle .
$$

Tracing (3.2)

$$
S=m \lambda-\Delta f
$$

so that

$$
\nabla \Delta f=m \nabla \lambda-\nabla S
$$

Inserting (3.12) into (3.11) and using (3.10)

$$
\begin{aligned}
\frac{1}{2} \Delta|\nabla f|^{2} & =|\operatorname{Hess}(f)|^{2}+\operatorname{Ric}(\nabla f, \nabla f)+m\langle\nabla \lambda, \nabla f\rangle-\langle\nabla S, \nabla f\rangle \\
& =|\operatorname{Hess}(f)|^{2}-\operatorname{Ric}(\nabla f, \nabla f)-(m-2)\langle\nabla \lambda, \nabla f\rangle .
\end{aligned}
$$

On the other hand, using

$$
\frac{1}{2}\left\langle\nabla|\nabla u|^{2}, X\right\rangle=\operatorname{Hess}(u)(\nabla u, X)
$$

and (3.2) from the above we obtain

$$
\begin{aligned}
\frac{1}{2} \Delta_{f}|\nabla f|^{2}= & \frac{1}{2} \Delta|\nabla f|^{2}-\frac{1}{2}\left\langle\nabla f, \nabla|\nabla f|^{2}\right\rangle \\
= & |\operatorname{Hess}(f)|^{2}-(m-2)\langle\nabla \lambda, \nabla f\rangle \\
& \quad-\operatorname{Ric}(\nabla f, \nabla f)-\operatorname{Hess}(f)(\nabla f, \nabla f) \\
= & |\operatorname{Hess}(f)|^{2}-\lambda|\nabla f|^{2}-(m-2)\langle\nabla \lambda, \nabla f\rangle,
\end{aligned}
$$

that is, (3.1)

\section{Corollary 3.2.}

$$
|\nabla f| \Delta_{f}|\nabla f| \geq-\lambda|\nabla f|^{2}-(m-2)\langle\nabla \lambda, \nabla f\rangle .
$$

Proof. From Kato's inequality

$$
|\operatorname{Hess}(f)|^{2} \geq|\nabla| \nabla f \|^{2} .
$$

Inserting into (3.1) we obtain (3.13).

We let $S$ denote the scalar curvature and $W$ the Weyl tensor of $(M,\langle\rangle$,$) .$ 
Lemma 3.3. Let $(M,\langle\rangle,, \nabla f)$ be a gradient Ricci almost soliton of dimension $m \geq 3$. Then

$$
\begin{aligned}
\Delta_{f} R_{i k}= & \Delta \lambda \delta_{i k}+(m-2) \lambda_{i k}+2 \lambda R_{i k}-\frac{2}{m-2}\left(|\mathrm{Ric}|^{2}-\frac{S^{2}}{m-1}\right) \delta_{i k} \\
& -\frac{2 m}{(m-1)(m-2)} S R_{i k}+\frac{4}{m-2} R_{i s} R_{s k}-2 W_{i j k s} R_{s j} .
\end{aligned}
$$

Therefore, tracing with respect to $i$ and $k$

$$
\frac{1}{2} \Delta_{f} S=\lambda S-|\operatorname{Ric}|^{2}+(m-1) \Delta \lambda .
$$

Remark 3.4. Note that for (3.15) we do not need the restriction $m \geq 3$. Indeed (3.15) can also be obtained by tracing (3.18) below for which it is not required $m \geq 3$.

Proof. It follows from (3.3) and the commutations relations $f_{i j k}-f_{i k j}=R_{l i j k} f_{l}$ that

$$
R_{i k, j}-R_{j k, i}=f_{s} R_{i j k s}+\lambda_{j} \delta_{k i}-\lambda_{i} \delta_{k j},
$$

and taking covariant derivatives we obtain the commutation relations

$$
R_{i k, j t}-R_{j k, i t}=f_{s t} R_{i j k s}+f_{s} R_{i j k s, t}+\lambda_{j t} \delta_{k i}-\lambda_{i t} \delta_{k j} .
$$

Also, from the commutation relations for the second covariant derivative of $R_{i k}$ we have

$$
R_{i j, k l}-R_{i j, l k}=R_{i t} R_{t j k l}+R_{j t} R_{t i k l},
$$

whence, contracting we obtain

$$
R_{j k, i j}=R_{j k, j i}+R_{s i} R_{s k}+R_{j i k s} R_{s j} .
$$

We now use (3.17) to obtain

$$
\Delta R_{i k}=R_{i k, j j}=R_{j k, i j}+f_{s} R_{i j k s, j}+f_{s j} R_{i j k s}+\Delta \lambda \delta_{k i}-\lambda_{i k} .
$$

On the other hand, from the second Bianchi identities we have

$$
f_{s} R_{i j k s, j}=R_{i k, s} f_{s}-R_{i s, k} f_{s}
$$

and inserting this into the above identity yields

$$
\Delta R_{i k}=f_{s j} R_{i j k s}-f_{s} R_{i s, k}+f_{s} R_{i k, s}+R_{j k, j i}+R_{s k} R_{i s}+R_{s j} R_{j i k s}+\Delta \lambda \delta_{i k}-\lambda_{i k} .
$$

Hence, from (3.5) and (3.2)

$$
\Delta R_{i k}=\frac{1}{2} S_{k i}+\lambda R_{i k}+R_{s k} R_{i s}+\Delta \lambda \delta_{i k}-\lambda_{i k}-2 R_{i j k s} R_{s j}-R_{i s, k} f_{s}+R_{i k, s} f_{s} .
$$


We shall now deal with the sum

$$
Z=\frac{1}{2} S_{k i}+R_{s k} R_{i s}-f_{s} R_{i s, k}
$$

Towards this aim we first observe that taking covariant derivative of (3.9) we have

$$
\frac{1}{2} S_{i k}=f_{j k} R_{i j}+f_{j} R_{i j, k}+(m-1) \lambda_{i k} .
$$

Substituting this into (3.19) and using the almost soliton equation (3.2) we obtain

$$
Z=(m-1) \lambda_{i k}+\lambda R_{i k}
$$

Substituting into (3.18) we therefore obtain

$$
\begin{aligned}
\Delta_{f} R_{i k} & =\Delta R_{i k}-f_{s} R_{i k, s} \\
& =2 \lambda R_{i k}+\Delta \lambda \delta_{i k}+(m-2) \lambda_{i k}-2 R_{i j k s} R_{j s} .
\end{aligned}
$$

The conclusion now follows recalling the decomposition of the curvature tensor into its irreducible components.

$$
\begin{aligned}
R_{i j k s}= & W_{i j k s}+\frac{1}{m-2}\left(R_{i k} \delta_{j s}-R_{i s} \delta_{j k}+R_{j s} \delta_{i k}-R_{j k} \delta_{i s}\right) \\
& -\frac{S}{(m-1)(m-2)}\left(\delta_{i k} \delta_{j s}-\delta_{i s} \delta_{j k}\right) .
\end{aligned}
$$

Substituting into (3.20) we obtain (3.14).

Corollary 3.5. Let $(M,\langle,\rangle \nabla f)$ be a conformally flat gradient Ricci almost soliton of dimension $m \geq 3$. Then

$$
\begin{aligned}
\Delta_{f} R_{i k}= & \Delta \lambda \delta_{i k}+(m-2) \lambda_{i k}+2 \lambda R_{i k}-\frac{2}{m-2}\left(|\mathrm{Ric}|^{2}-\frac{S^{2}}{m-1}\right) \delta_{i k} \\
& -\frac{2 m}{(m-1)(m-2)} S R_{i k}+\frac{4}{m-2} R_{i s} R_{s k} .
\end{aligned}
$$

Corollary 3.6. Let $(M,\langle\rangle,, \nabla f)$ be as in Corollary 3.5 and let $T=\operatorname{Ric}-\frac{S}{m}\langle$, be the trace free Ricci tensor. Then

$$
\begin{aligned}
\frac{1}{2} \Delta_{f}|T|^{2}= & |\nabla T|^{2}+2\left(\lambda-S \frac{m-2}{m(m-1)}\right)|T|^{2} \\
& +(m-2)\langle\operatorname{Hess}(\lambda), T\rangle+\frac{4}{m-2} \operatorname{tr}\left(T^{3}\right),
\end{aligned}
$$

with $T^{3}=T \circ T \circ T$. In particular, using Okumura's lemma

$$
\begin{aligned}
\frac{1}{2} \Delta_{f}|T|^{2} \geq & 2\left(\lambda-S \frac{m-2}{m(m-1)}\right)|T|^{2}-\frac{4}{\sqrt{m(m-1)}}|T|^{3} \\
& +(m-2)\langle\operatorname{Hess}(\lambda), T\rangle .
\end{aligned}
$$


Proof. We compute

$$
\begin{aligned}
\Delta_{f}|T|^{2} & =2|\nabla T|^{2}+2\langle T, \Delta T\rangle-\left\langle\nabla f, \nabla|T|^{2}\right\rangle \\
& =2 T_{i k, l} T_{i k, l}+2 T_{i k} \Delta_{f} T_{i k} .
\end{aligned}
$$

Using equation (3.15) and the definition of $T$, we have

$$
\begin{aligned}
\Delta_{f} T_{i k}= & \Delta_{f} R_{i k}-\frac{1}{m} \delta_{i k} \Delta_{f} S=\Delta \lambda \delta_{i k}+(m-2) \lambda_{i k}+2 \lambda R_{i k} \\
& -\frac{2}{m-2}|\mathrm{Ric}|^{2} \delta_{i k}+\frac{2}{(m-2)(m-1)} S^{2} \delta_{i k}-\frac{2 m}{(m-1)(m-2)} S R_{i k} \\
& +\frac{4}{m-2} R_{i s} R_{s k}-\frac{2}{m} \lambda S \delta_{i k}+\frac{2}{m}|\mathrm{Ric}|^{2} \delta_{i k}-\frac{2}{m}(m-1) \Delta \lambda \delta_{i k} \\
= & -\frac{m-2}{m} \delta_{i k} \Delta \lambda+(m-2) \lambda_{i k}+2 \lambda T_{i k}-\frac{2 m S}{(m-1)(m-2)} T_{i k} \\
& -\frac{4}{m(m-2)} \delta_{i k}|\operatorname{Ric}|^{2}+\frac{4}{m-2}\left(T_{i s} T_{s k}+\frac{S^{2}}{m^{2}} \delta_{i k}+\frac{2 S}{m} T_{i k}\right) .
\end{aligned}
$$

Thus, recalling that $T$ is trace free, we obtain

$$
\begin{aligned}
T_{i k} \Delta_{f} T_{i k}= & 2 \lambda|T|^{2}+(m-2) \lambda_{i k} T_{i k} \\
& -\frac{2(m-2) S}{m(m-1)}|T|^{2}+\frac{4}{m-2} \operatorname{tr}\left(T^{3}\right),
\end{aligned}
$$

and (3.21) follows. Inequality (3.22) follows immediately, since by Okumura's Lemma, [19],

$$
\operatorname{tr}\left(T^{3}\right) \geq-\frac{m-2}{\sqrt{m(m-1)}}|T|^{3} .
$$

\section{Volume comparison results}

In order to prove Theorem 1.2 we need two auxiliary results. The first is an improvement of Theorem 1.2 (a) of Wei and Wylie [31], where they assume $\theta$ and $G$ below to be constant.

Theorem 4.1. Let $\left(M,\langle\rangle,, e^{-f} d \mathrm{vol}\right)$ be a complete weighted manifold such that

$$
\langle\nabla r, \nabla f\rangle \geq-\theta(r)
$$

for some non-decreasing function $\theta \in C^{0}\left(\mathbb{R}_{0}^{+}\right)$. Assume

$$
\operatorname{Ric}_{f} \geq-(m-1) G(r)\langle,\rangle
$$


for a smooth positive function $G$ on $\mathbb{R}_{0}^{+}$, even at the origin. Let $g$ be a solution on $\mathbb{R}_{0}^{+}$of

$$
\left\{\begin{array}{l}
g^{\prime \prime}-G g \geq 0 \\
g(0)=0, \quad g^{\prime}(0) \geq 1 .
\end{array}\right.
$$

Then there exists a constant $D>0$ such that $\forall r \geq 0$

$$
\operatorname{vol}_{f}\left(B_{r}\right) \leq D \int_{0}^{r} g(t)^{m-1} e^{\int_{0}^{t} \theta(s) d s} d t .
$$

Proof. Let $h$ be the solution on $\mathbb{R}_{0}^{+}$of the Cauchy problem

$$
\left\{\begin{array}{l}
h^{\prime \prime}-G h=0 \\
h(0)=0, \quad h^{\prime}(0)=1 .
\end{array}\right.
$$

Note that $h>0$ on $\mathbb{R}^{+}$since $G \geq 0$. Fix $x \in M \backslash$ (cut $\left.(o) \cup\{o\}\right)$ and let $\gamma$ : $[0, l] \rightarrow M, l=$ length $(\gamma)$, be a minimizing geodesic with $\gamma(0)=o, \gamma(l)=x$. Note that $G(r \circ \gamma)(t)=G(t)$. From the Bochner formula applied to the distance function $r$ we have

$$
0=|\operatorname{Hess}(r)|^{2}+\langle\nabla r, \nabla \Delta r\rangle+\operatorname{Ric}(\nabla r, \nabla r)
$$

so that, using the Schwarz inequality, it follows that the function $\varphi(t)=(\Delta r) \circ$ $\gamma(t), t \in(0, l]$, satisfies the Riccati inequality

$$
\varphi^{\prime}+\frac{1}{m-1} \varphi^{2} \leq-\operatorname{Ric}(\nabla r \circ \gamma, \nabla r \circ \gamma)
$$

on $(0, l]$. With $h$ as in (4.5) and using the definition of $\operatorname{Ric}_{f}$, (4.2) and (4.7) we compute

$$
\begin{aligned}
\left(h^{2} \varphi\right)^{\prime}= & 2 h h^{\prime} \varphi+h^{2} \varphi^{\prime} \\
\leq & 2 h h^{\prime} \varphi-\frac{h^{2} \varphi^{2}}{m-1}+(m-1) G(t) h^{2}+\operatorname{Hess}(f)(\nabla r \circ \gamma, \nabla r \circ \gamma) h^{2} \\
= & -\left(\frac{h \varphi}{\sqrt{m-1}}-\sqrt{m-1} h^{\prime}\right)^{2}+(m-1)\left(h^{\prime}\right)^{2}+(m-1) G(t) h^{2} \\
& +h^{2}(f \circ \gamma)^{\prime \prime} .
\end{aligned}
$$

We let

$$
\varphi_{G}(t)=(m-1) \frac{h^{\prime}}{h}(t)
$$

so that, using (4.5)

$$
\left(h^{2} \varphi_{G}\right)^{\prime}=(m-1)\left(h^{\prime}\right)^{2}+(m-1) G(t) h^{2} .
$$


Inserting into the above inequality we obtain

$$
\left(h^{2} \varphi\right)^{\prime} \leq\left(h^{2} \varphi_{G}\right)^{\prime}+h^{2}(f \circ \gamma)^{\prime \prime} .
$$

Integrating (4.8) on $[0, r]$ and using (4.5) yields

$$
h^{2}(r) \varphi(r) \leq h^{2}(r) \varphi_{G}(r)+\int_{0}^{r} h^{2}(f \circ \gamma)^{\prime \prime} d t .
$$

Next we recall that

$$
\varphi_{f}=\left(\Delta_{f} r\right) \circ \gamma=(\Delta r) \circ \gamma-\langle\nabla f, \nabla r\rangle \circ \gamma=\varphi-(f \circ \gamma)^{\prime} .
$$

Thus, using (4.9), (4.5) and integrating by parts we compute

$$
\begin{aligned}
h^{2} \varphi_{f} & \leq h^{2} \varphi_{G}-h^{2}(f \circ \gamma)^{\prime}+\int_{0}^{r} h^{2}(f \circ \gamma)^{\prime \prime} d t \\
& =h^{2} \varphi_{G}-h^{2}(f \circ \gamma)^{\prime}+\left.\left(h^{2}(f \circ \gamma)^{\prime}\right)\right|_{0} ^{r}-\int_{0}^{r}\left(h^{2}\right)^{\prime}(f \circ \gamma)^{\prime} d t \\
& =h^{2} \varphi_{G}-\int_{0}^{r}\left(h^{2}\right)^{\prime}(f \circ \gamma)^{\prime} d t,
\end{aligned}
$$

that is,

$$
h^{2} \varphi_{f} \leq h^{2} \varphi_{G}-\int_{0}^{r}\left(h^{2}\right)^{\prime}(f \circ \gamma)^{\prime} d t
$$

on $(0, l]$. We observe that, because of (4.5) and $G \geq 0,\left(h^{2}\right)^{\prime}=2 h h^{\prime} \geq 0$ so that, using (4.1), (4.5) and the monotonicity of $\theta,(4.11)$ yields

$$
h^{2} \varphi_{f} \leq h^{2} \varphi_{G}+\theta(r) h^{2}
$$

on $(0, l]$, and

$$
\varphi_{f} \leq \varphi_{G}+\theta(r)
$$

on $(0, l]$. In particular

$$
\Delta_{f} r(x) \leq(m-1) \frac{h^{\prime}(r(x))}{h(r(x))}+\theta(r(x))
$$

on $M \backslash(\{o\} \cup \operatorname{cut}(o))$. Proceeding as in [26, Theorem 2.4] one shows that (4.12) holds weakly on all of $M$ and reasoning as in [26, Theorem 2.14] one shows that

$$
\operatorname{vol}_{f}\left(\partial B_{r}\right) \leq D h(r)^{m-1} e^{\int_{0}^{r} \theta(t) d t}
$$

for some constant $D>0$. Integrating over $[0, r]$ and using the co-area formula we get

$$
\operatorname{vol}_{f}\left(B_{r}\right) \leq D \int_{0}^{r} h(t)^{m-1} e^{\int_{0}^{t} \theta(s) d s} d t .
$$

Since $g$ in (4.3) is a subsolution of (4.5) it follows, in [26, Lemma 2.1], that $h \leq g$ on $\mathbb{R}_{0}^{+}$so that (4.14) immediately implies (4.4) 
A second estimate on $\varphi_{f}$ can also be derived, replacing assumption (4.1) with

$$
\xi(r) \leq f \leq \omega(r),
$$

for some functions $\omega, \xi \in C^{1}\left(\mathbb{R}_{0}^{+}\right)$with $\omega$ non decreasing and such that $\xi^{\prime}(r) \leq$ $\omega^{\prime}(r)$.

Towards this aim we integrate (4.11) again by parts to obtain

$$
h^{2} \varphi_{f} \leq h^{2} \varphi_{G}-\left.\left[\left(h^{2}\right)^{\prime}(f \circ \gamma)\right]\right|_{0} ^{r}+\int_{0}^{r}\left(h^{2}\right)^{\prime \prime}(f \circ \gamma) d t .
$$

Now, using (4.5),

$$
\left(h^{2}\right)^{\prime \prime}=2\left(h^{\prime}\right)^{2}+2 G h^{2} \geq 0,
$$

because of the sign of $G$. Thus using (4.15), (4.5) and the fact that $\omega$ is nondecreasing, from the above we obtain

$$
\begin{aligned}
h^{2} \varphi_{f} & \leq h^{2} \varphi_{G}-\left.\left(h^{2}\right)^{\prime}(f \circ \gamma)\right|_{0} ^{r}+\left.\omega(r)\left(h^{2}\right)^{\prime}\right|_{0} ^{r} \\
& \leq h^{2} \varphi_{G}-\left(h^{2}\right)^{\prime}(r)(f \circ \gamma)(r)+\left(h^{2}\right)^{\prime}(r) \omega(r) \\
& \leq h^{2} \varphi_{G}+\left(h^{2}\right)^{\prime}(r)[\omega(r)-(f \circ \gamma)(r)] .
\end{aligned}
$$

Now

$$
\left(h^{2}\right)^{\prime}=2 h h^{\prime}=\frac{2}{m-1} h^{2}(m-1) \frac{h^{\prime}}{h}=\frac{2}{m-1} h^{2} \varphi_{G}, r>0
$$

so that the above inequality may be rewritten as

$$
h^{2} \varphi_{f} \leq h^{2}\left(1+\frac{2}{m-1}(\omega(r)-(f \circ \gamma)(r))\right) \varphi_{G}, r>0
$$

and using (4.15)

$$
\varphi_{f} \leq\left(1+\frac{2}{m-1}(\omega(r)-\xi(r))\right) \varphi_{G}, r>0 .
$$

Let $\tilde{\omega}(r) \geq \omega(r)-\xi(r) \geq 0$. Similarly to what we did in Theorem 4.1 we arrive at (4.13), where $\theta(t)$ is now substituted by $\frac{2}{m-1} \tilde{\omega}(t) \varphi_{G}(t)$. Thus we need to estimate $e^{\int_{r_{0}}^{r} 2 \tilde{\omega}(t) \frac{h^{\prime}}{h}}$.

$$
\begin{aligned}
\int_{r_{0}}^{r} \frac{2}{m-1} \tilde{\omega}(t) \frac{h^{\prime}}{h}= & \frac{2}{m-1} \tilde{\omega}(r) \log h^{m-1}(r)-\frac{2}{m-1} \tilde{\omega}\left(r_{0}\right) \log h^{m-1}\left(r_{0}\right) \\
& -\int_{r_{0}}^{r} \frac{2}{m-1} \tilde{\omega}^{\prime}(t) \log h^{m-1}(t) d t .
\end{aligned}
$$


Now, by (4.5), $h(t) \nearrow+\infty$ as $t \rightarrow+\infty$. Choose $r_{0}$ sufficiently large that $h\left(r_{0}\right) \geq 1$. Since $\tilde{\omega}^{\prime} \geq 0$

$$
\int_{r_{0}}^{r} \frac{2}{m-1} \tilde{\omega}(t)\left(\log h^{m-1}\right)^{\prime} d t \leq \log (h(r))^{2 \tilde{\omega}(r)}-A,
$$

and

$$
e^{\int_{r_{0}}^{r} \frac{2}{m-1} \tilde{\omega}(t) \varphi_{G}} \leq h(r)^{2 \tilde{\omega}(r)} e^{-A}
$$

Hence, from (4.13),

$$
\operatorname{vol}_{f}\left(\partial B_{r}\right) \leq D h(r)^{m-1+2 \tilde{\omega}(r)} .
$$

Since $h \leq g$ we have thus proven the following result, which improves on [31, Theorem 1.2 (b)] of Wei and Wylie.

Theorem 4.2. Let $\left(M,\langle\rangle,, e^{-f} d \mathrm{vol}\right)$ be a complete weighted manifold such that

$$
\xi(r) \leq f \leq \omega(r)
$$

for some functions $\omega, \xi \in C^{1}\left(\mathbb{R}_{0}^{+}\right)$with $\omega$ non decreasing and such that $\xi^{\prime}(r) \leq$ $\omega^{\prime}(r)$. Assume

$$
\operatorname{Ric}_{f} \geq-(m-1) G(r)\langle,\rangle
$$

for a smooth positive function $G$ on $\mathbb{R}_{0}^{+}$, even at the origin.

Let $\tilde{\omega}(r)=\omega(r)-\xi(r)$ and $g$ be a solution on $\mathbb{R}_{0}^{+}$of

$$
\left\{\begin{array}{l}
g^{\prime \prime}-G g \geq 0 \\
g(0)=0, \quad g^{\prime}(0) \geq 1 .
\end{array}\right.
$$

Then there exist constants $C, B>0$ such that, $\forall r \geq r_{0}>0$,

$$
\operatorname{vol}_{f}\left(B_{r}\right) \leq C+B \int_{r_{0}}^{r} g(t)^{(m-1)+2 \tilde{\omega}(t)} d t
$$

We end this discussion with the following simple proposition which marginally extends a previous result by Wei and Wylie, [31], and which will be used in the proof of Theorem 1.4.

Proposition 4.3. Let $\left(M,\langle\rangle,, e^{-f}\right)$ be a weighted manifold and assume that

$$
\operatorname{Ric}_{f} \geq D(1+r)^{-\mu} .
$$

(i) If $D>0$ and $0 \leq \mu \leq 1$, then there exist constants $C_{j}>0$ such that for every $r>2$,

$$
\operatorname{vol}_{f}\left(\partial B_{r}\right) \leq\left\{\begin{array}{ll}
C_{1} e^{-C_{2} r \log (1+r)} & \text { if } \quad \mu=1 \\
C_{1} e^{-C_{2} r^{2-\mu}} & \text { if } \quad 0 \leq \mu<1
\end{array} \text { and } \operatorname{vol}_{f}\left(B_{r}\right) \leq C_{3}\right.
$$


(ii) If $D=0$ then there exist constants $C_{j}>0$ such that for every $r>2$,

$$
\operatorname{vol}_{f}\left(\partial B_{r}\right) \leq C_{1} e^{r} \text { and } \operatorname{vol}_{f}\left(B_{r}\right) \leq C_{2} e^{r} .
$$

(iii) If $D<0$ then there exist constants $C_{j}>0$ such that for every $r>2$,

$$
\operatorname{vol}_{f}\left(\partial B_{r}\right) \leq\left\{\begin{array}{lll}
C_{1} e^{C_{2} r} & \text { if } \quad \mu>1 \\
C_{1} e^{C_{2} r \log r} & \text { if } & \mu=1 \\
C_{1} e^{C_{2} r^{2-\mu}} & \text { if } & 0 \leq \mu<1
\end{array}\right.
$$

and

$$
\operatorname{vol}_{f}\left(B_{r}\right) \leq \begin{cases}C_{3} e^{C_{2} r} & \text { if } \mu>1 \\ C_{3}(\log r)^{-1} e^{C_{2} r \log r} & \text { if } \mu=1 \\ C_{3} r^{\mu-1} e^{C_{2} r^{2-\mu}} & \text { if } 0 \leq \mu<1\end{cases}
$$

Proof. Maintaining the notation introduced above, it follows from (4.7), (4.10) and the definition of $\operatorname{Ric}_{f}$ that if $\varphi_{f}=\Delta_{f} r \circ \gamma$ then

$$
\varphi_{f}^{\prime}=\varphi^{\prime}-(f \circ \gamma)^{\prime \prime} \leq-\frac{\varphi^{2}}{m-1}-\operatorname{Ric}(\dot{\gamma}, \dot{\gamma})-\operatorname{Hess} f(\dot{\gamma}, \dot{\gamma}) \leq-\operatorname{Ric}_{f}(\dot{\gamma}, \dot{\gamma}) .
$$

Thus, if we assume that $\operatorname{Ric}_{f} \geq \theta(r(x))$ and that the ball $B_{\varepsilon}$ is contained in the domain of the normal coordinates at $o$, setting $C=\max _{\partial B_{\varepsilon}} \Delta_{f} r$ and integrating between $\varepsilon$ and $r(x)$ we obtain

$$
\Delta_{f} r(x) \leq C-\int_{\varepsilon}^{r(x)} \theta(t) d t
$$

pointwise in the $M \backslash\left(B_{\varepsilon} \cup \operatorname{cut}(o)\right)$ and weakly on $M \backslash B_{\varepsilon}$. From this, arguing as in $[26$, Theorem 2.4] we deduce that

$$
\operatorname{vol}_{f}\left(\partial B_{r}\right) \leq e^{C\left(r-r_{o}\right)-\int_{r_{o}}^{r}\left(\int_{\varepsilon}^{t} \theta(s) d s\right) d t} \operatorname{vol}_{f}\left(\partial B_{r_{o}}\right) .
$$

The conclusion now follows estimating the integral on the right hand side for $\theta(s)=D(1+s)^{-\mu}$.

The second ingredient we shall need in the proof of Theorem 1.2 is the following version of [14, Theorem 5.2].

Theorem 4.4. Let $\left(M,\langle\rangle,, e^{-f} d \mathrm{vol}\right)$ be a complete weighted manifold. Given $\sigma, \mu \in \mathbb{R}$, let $v=\mu+2(\sigma-1)$ and assume that $\sigma \geq 0, \sigma-v>0$. Let $u \in C^{1}(M)$ be a function such that

$$
\hat{u}=\limsup _{r(x) \rightarrow+\infty} \frac{u(x)}{r(x)^{\sigma}}<+\infty
$$


and suppose that

$$
\liminf _{r \rightarrow+\infty} \frac{\log \operatorname{vol}_{f}\left(B_{r}\right)}{r^{\sigma-v}}=d_{0}<+\infty .
$$

Then given $\gamma \in \mathbb{R}$ such that

$$
\Omega_{\gamma}=\{x \in M: u(x)>\gamma\} \neq \varnothing
$$

we have

$$
\inf _{\Omega_{\gamma}}(1+r(x))^{\mu} \Delta_{f} u \leq C \max \{\hat{u}, 0\}
$$

with

$$
C=\left\{\begin{array}{lll}
0 & \text { if } & \sigma=0 \\
d_{0}(\sigma-v)^{2} & \text { if } & 0<v<\sigma \\
d_{0} \sigma(\sigma-v) & \text { if } & \sigma>0, v \geq \sigma .
\end{array}\right.
$$

We are now ready to give the:

Proof of Theorem 1.2. First of all from Lemma 3.1 and assumption (1.6) we know that $|\nabla f|^{2}$ satisfies the differential inequality

$$
\Delta_{f}|\nabla f|^{2} \geq-2 \lambda|\nabla f|^{2}
$$

on $M$. Furthermore, from (1.4) we deduce

$$
\langle\nabla r, \nabla f\rangle \geq-a(1+r)^{\frac{\sigma}{2}},
$$

for some constant $a>0$. Using (1.5) we apply Theorem 4.1 with the choice $\theta(r)=$ $a(1+r)^{\frac{\sigma}{2}}$ to obtain

$$
\operatorname{vol}_{f}\left(B_{r}\right) \leq D \int_{0}^{r} g(t)^{m-1} e^{\frac{2 a}{(\sigma+2)(m-1)}(1+t)^{\frac{\sigma+2}{2}}} d t
$$

for some constant $B>0$ and where $g$ solves (4.5) with $G(r)=B^{2}\left(1+r^{2}\right)^{\frac{\alpha}{2}}$. By [26, Proposition 2.11] it follows that, for $r \gg 1$,

$$
g(r) \leq C_{1} \exp \left(C_{2} e^{\frac{\alpha+2}{2}}\right),
$$

for some constants $C_{1}, C_{2}$. Thus, a simple computation shows that

$$
\frac{\log \operatorname{vol}_{f}\left(B_{r}\right)}{r^{2-\mu-\sigma}} \leq C\left(r^{\mu+\sigma-1+\frac{\alpha}{2}}+r^{\mu-1+\frac{3}{2} \sigma}\right)
$$

for $r \gg 1$ and some constant $C>0$. Using (1.3) we see that assumption (4.16) of Theorem 4.4 is satisfied with $v=\mu+2(\sigma-1)$ so that $\sigma-v=2-\mu-\sigma$. On the other hand, from (4.17) and (1.5) we have

$$
(1+r(x))^{\mu} \Delta_{f}|\nabla f|^{2} \geq H|\nabla f|^{2}
$$


for some appropriate constant $H>0$. Assume that $|\nabla f|$ is different from 0 and choose $\gamma>0$ so that

$$
\Omega_{\gamma}=\left\{x \in M:|\nabla f|^{2}>\gamma\right\} \neq \emptyset .
$$

From (1.4), (4.18) and Theorem 4.4 we immediately obtain a contradiction.

\section{A weighted Omori-Yau maximum principle}

Theorem 4.4 stated in the previous section represents a refined and generalized version of what is known in the literature as the weak maximum principle at infinity; [24,25]. Indeed, taking $\sigma=\mu=0$, we deduce that, for a smooth function $u$ on $M$ satisfying $\sup _{M} u=u^{*}<+\infty$, there exists a sequence $\left\{x_{n}\right\}$ along which

$$
u\left(x_{n}\right)>u^{*}-\frac{1}{n}, \text { and } \Delta_{f} u\left(x_{n}\right)<\frac{1}{n} .
$$

In general, under volume growth conditions, nothing can be said about the behavior of the gradient of $u$. If, along the same sequence $\left\{x_{n}\right\}$, we have that

$$
\left|\nabla u\left(x_{n}\right)\right|<\frac{1}{n}
$$

then we say that the full Omori-Yau maximum principle for the $f$-Laplacian holds. The following result, which is a generalization of [25, Theorem 1.9], gives functiontheoretic sufficient conditions for a weighted Riemannian manifold $\left(M,\langle\rangle,, e^{-f} d\right.$ vol) to satisfy the Omori-Yau maximum principle.

Theorem 5.1. Let $\left(M,\langle\rangle,, e^{-f} d \mathrm{vol}\right)$ be a weighted Riemannian manifold and assume that there exists a non-negative $C^{2}$ function $\gamma$ satisfying the following conditions

$$
\begin{gathered}
\gamma(x) \rightarrow+\infty \text { as } x \rightarrow \infty \\
\exists A>0 \text { such that }|\nabla \gamma| \leq A \gamma^{\frac{1}{2}} \text { off a compact set } \\
\exists B>0 \text { such that } \Delta_{f} \gamma \leq B \gamma^{\frac{1}{2}} G\left(\gamma^{\frac{1}{2}}\right)^{\frac{1}{2}} \text { off a compact set }
\end{gathered}
$$

where $G$ is a smooth function on $[0,+\infty)$ satisfying

$$
\begin{array}{ll}
\text { (i) } G(0)>0 & \text { (ii) } G^{\prime}(t) \geq 0 \text { on }[0,+\infty) \\
\text { (iii) } G(t)^{-\frac{1}{2}} \notin L^{1}(+\infty) & \text { (iv) } \limsup _{t \rightarrow+\infty} \frac{t G\left(t^{\frac{1}{2}}\right)}{G(t)}<+\infty
\end{array}
$$

Then, given any function $u \in C^{2}(M)$ with $u^{*}=\sup _{M} u<+\infty$, there exists a sequence $\left\{x_{n}\right\}_{n} \subset M$ such that
(i) $u\left(x_{k}\right)>u^{*}-\frac{1}{k}$;
(ii) $\left|\nabla u\left(x_{k}\right)\right|<\frac{1}{k}$;
(iii) $\Delta_{f} u\left(x_{k}\right)<\frac{1}{k}$; 
for each $k \in \mathbb{N}$, i.e. the Omori-Yau maximum principle for $\Delta_{f}$ holds on $\left(M,\langle\rangle,, e^{-f} d \mathrm{vol}\right)$.

The proof of this theorem is similar to that of [25, Theorem 1.9] and we refer to this one for more details.

Proof. We define the function

$$
\varphi(t)=e^{\int_{0}^{t} G(s)^{-\frac{1}{2}} d s} .
$$

Proceeding as in [25] and using assumption (5.4) (iv), we have that

$$
0 \leq \frac{\varphi^{\prime}(t)}{\varphi(t)}<c\left(t G\left(t^{\frac{1}{2}}\right)\right)^{-\frac{1}{2}}
$$

for some constant $c>0$. Next, we fix a point $p \in M$ and, $\forall k \in \mathbb{N}$, we define

$$
F_{k}(x)=\frac{u(x)-u(p)+1}{\varphi(\gamma(x))^{\frac{1}{k}}} .
$$

Then $F_{k}(p)=1 / \varphi(\gamma(p))^{1 / k}>0$. Moreover, since $u^{*}<+\infty$ and $\varphi(\gamma(x)) \rightarrow$ $+\infty$ as $x \rightarrow+\infty$, we have $\lim \sup _{x \rightarrow \infty} F_{k}(x) \leq 0$. Thus, $F_{k}$ attains a positive absolute maximum at $x_{k} \in M$. Iterating this procedure, we produce a sequence $\left\{x_{k}\right\}$. It is shown in [25] that

$$
\limsup _{k \rightarrow+\infty} u\left(x_{k}\right)=u^{*}
$$

and by passing to a subsequence if necessary, we may assume that

$$
\lim _{k \rightarrow+\infty} u\left(x_{k}\right)=u^{*}
$$

If $\left\{x_{k}\right\}$ remains in a compact set, then $x_{k} \rightarrow \bar{x}$ as $k \rightarrow+\infty$ and the sequence $z_{k}=\bar{x}$, for each $k$, clearly satisfies (5.5). We only need to consider the case when $x_{k} \rightarrow \infty$ so that, according to (5.1), $\gamma\left(x_{k}\right) \rightarrow+\infty$. Since $F_{k}$ attains a positive maximum at $x_{k}$ we have

$$
\text { (i) }\left(\nabla \log F_{k}\right)\left(x_{k}\right)=0 ; \quad \text { (ii) } \Delta_{f}\left(\log F_{k}\right)\left(x_{k}\right)=\Delta\left(\log F_{k}\right)\left(x_{k}\right) \leq 0 \text {. }
$$

Note that from (5.7)(i) we have that

$$
\nabla u\left(x_{k}\right)=\frac{1}{k}\left(u\left(x_{k}\right)-u(p)+1\right) \frac{\varphi^{\prime}\left(\gamma\left(x_{k}\right)\right)}{\varphi\left(\gamma\left(x_{k}\right)\right)} \nabla \gamma\left(x_{k}\right) .
$$


Furthermore, reasoning as in [25] we have

$$
\begin{aligned}
\Delta u\left(x_{k}\right) \leq \frac{u\left(x_{k}\right)-u(p)+1}{k} & \left\{\frac{\varphi^{\prime}\left(\gamma\left(x_{k}\right)\right)}{\varphi\left(\gamma\left(x_{k}\right)\right)} \Delta(\gamma)\left(x_{k}\right)\right. \\
& \left.+\frac{1}{k}\left(\frac{\varphi^{\prime}\left(\gamma\left(x_{k}\right)\right)}{\varphi\left(\gamma\left(x_{k}\right)\right)}\right)^{2}\left|\nabla \gamma\left(x_{k}\right)\right|^{2}\right\} .
\end{aligned}
$$

Assume now that (5.2) and (5.3) hold so that they hold at $x_{k}$ for sufficiently large $k$. A computation shows that

$$
\left|\nabla u\left(x_{k}\right)\right| \leq \frac{a}{k} \cdot \frac{u\left(x_{k}\right)-u(p)+1}{G\left(\gamma\left(x_{k}\right)^{1 / 2}\right)^{1 / 2}}
$$

for some costant $a>0$. Therefore, using (5.3) and (5.6), we obtain

$$
\begin{aligned}
& \Delta_{f} u\left(x_{k}\right)=\Delta u\left(x_{k}\right)-\langle\nabla u, \nabla f\rangle\left(x_{k}\right) \\
& \leq \frac{u\left(x_{k}\right)-u(p)+1}{k}\left\{\frac{\varphi^{\prime}\left(\gamma\left(x_{k}\right)\right)}{\varphi\left(\gamma\left(x_{k}\right)\right)} \Delta \gamma\left(x_{k}\right)+\frac{1}{k}\left(\frac{\varphi^{\prime}\left(\gamma\left(x_{k}\right)\right)}{\varphi\left(\gamma\left(x_{k}\right)\right)}\right)^{2}|\nabla \gamma|^{2}\left(x_{k}\right)\right. \\
&\left.\quad-\frac{\varphi^{\prime}\left(\gamma\left(x_{k}\right)\right)}{\varphi\left(\gamma\left(x_{k}\right)\right)}\langle\nabla \gamma, \nabla f\rangle\left(x_{k}\right)\right\} \\
&=\frac{u\left(x_{k}\right)-u(p)+1}{k}\left\{\frac{\varphi^{\prime}\left(\gamma\left(x_{k}\right)\right)}{\varphi\left(\gamma\left(x_{k}\right)\right)} \Delta_{f} \gamma\left(x_{k}\right)+\frac{1}{k}\left(\frac{\varphi^{\prime}\left(\gamma\left(x_{k}\right)\right)}{\varphi\left(\gamma\left(x_{k}\right)\right)}\right)^{2}|\nabla \gamma|^{2}\left(x_{k}\right)\right\} \\
& \leq \frac{u\left(x_{k}\right)-u(p)+1}{k}\left\{\frac{c}{\gamma^{1 / 2} G\left(\gamma^{1 / 2}\right)^{1 / 2}} B \gamma^{1 / 2} G\left(\gamma^{1 / 2}\right)^{1 / 2}+\frac{1}{k} \cdot \frac{c^{2}}{\gamma G\left(\gamma^{1 / 2}\right)} A^{2} \gamma\right\}
\end{aligned}
$$

and the RHS tends to zero as $k \rightarrow+\infty$.

We now consider two important situations where Theorem 5.1 applies. First, we recover a result by M. Fernández-López and E. García Río, [6].

Corollary 5.2. Let $(M,\langle\rangle,, \nabla f)$ be a gradient shrinking Ricci soliton. Then, the Omori-Yau maximum principle for the $f$-Laplacian holds.

Proof. Set $G(t)=t^{2}+1$ and $\gamma=f$. It is proved in [6] that

$$
\begin{gathered}
\gamma \rightarrow+\infty \text { as } x \rightarrow \infty \\
|\nabla f|=|\nabla \gamma| \leq \sqrt{f}=\sqrt{\gamma} \\
\Delta_{f} \gamma=\Delta f-|\nabla f|^{2} \leq \Delta f \leq \gamma^{1 / 2} G\left(\gamma^{1 / 2}\right)^{1 / 2},
\end{gathered}
$$

that is, conditions (5.1), (5.2) and (5.3) of Theorem 5.1 are satisfied. 
The second situation concerns with general weighted manifolds whose BakryEmery Ricci tensor is suitably controlled. Clearly it represents an extension of Corollary 5.2 to non necessarily shrinking almost solitons.

Corollary 5.3. Let $\left(M,\langle\rangle,, e^{-f} d \mathrm{vol}\right)$ be a complete weighted manifold such that

$$
\operatorname{Ric}_{f} \geq-(m-1) G(r)\langle,\rangle
$$

for a smooth positive function $G$ satisfyng (5.4), even at the origin. Assume also that

$$
|\nabla f| \leq C G(r)^{1 / 2} .
$$

Then the Omori-Yau maximum principle for the $f$-Laplacian holds on $M$.

Proof. Let $h$ be as in Theorem 4.1. Then

$$
\Delta_{f} r \leq(m-1) \frac{h^{\prime}}{h}+|\nabla r||\nabla f| \leq(m-1) \frac{h^{\prime}}{h}+C G(r)^{1 / 2} \leq D G(r)^{1 / 2},
$$

and thus

$$
\Delta_{f} r^{2}=2+2 r \Delta_{f} r \leq 2+2 r G(r)^{1 / 2} \leq \operatorname{Cr} G(r)^{1 / 2},
$$

and the hypotheses of Theorem 5.1 are satisfied with $\gamma=r^{2}$ outside the cut-locus of $o$. This is enough to conclude. Indeed, since $G$ is non-decreasing, we can use the Calabi trick to show that, without loss of generality, the sequence $\left\{x_{k}\right\}$ constructed in the proof of Theorem 5.1 can be always taken in $M \backslash \operatorname{cut}(o)$.

\section{Triviality in the presence of weighted Poincaré-Sobolev inequalities}

This section aims to prove Theorem 1.6 of the Introduction. In a sense it can be considered as an isolation result for the soliton function of almost solitons with $L^{p}$ soliton structure. Again we need a preliminary result. The next proposition can be deduced by simple modifications to the proof of [26, Theorem 9.12].

Proposition 6.1. Let $\left(M,\langle\rangle,, e^{-f} d \mathrm{vol}\right)$ be a complete weighted manifold and assume that, for some $0 \leq \alpha<1$, the Poincaré-Sobolev inequality

$$
\int_{M}|\nabla \varphi|^{2} e^{-f} d \mathrm{vol} \geq S(\alpha)^{-1}\left\{\int_{M}|\varphi|^{\frac{2}{1-\alpha}} e^{-f} d \mathrm{vol}\right\}^{1-\alpha},
$$

holds for all $\varphi \in C_{0}^{\infty}(M)$ and some constant $S(\alpha)>0$. Let $B \in \mathbb{R}, q(x) \in$ $C^{0}(M)$ and let $\psi \in \operatorname{Lip}_{\text {loc }}(M)$ be a non-negative weak solution of

$$
\psi \Delta_{f} \psi+q(x) \psi^{2} \geq-B|\nabla \psi|^{2}
$$

on M. Assume that, for some

$$
\sigma>\max \{1, B+1\}
$$


we have

$$
\int_{B_{r}} \psi^{\sigma} e^{-f}=o\left(r^{2}\right)
$$

as $r \rightarrow+\infty$. Then either $\psi \equiv 0$ or otherwise

$$
\left\|q_{+}(x)\right\|_{L^{\frac{1}{\alpha}}\left(M, e^{-f}\right)} \geq \frac{4}{S(\alpha)} \frac{p-1}{p^{2}} .
$$

Remark 6.2. As we already observed in the Introduction, the validity of a PoincaréSobolev inequality forces $M$ to have infinite weighted volume. Furthermore, if $\alpha=0,(6.1)$ means precisely that the manifold has positive weighted spectral radius.

An immediate consequence of Proposition 6.1 is the following:

Theorem 6.3. Let $(M,\langle\rangle,, \nabla f)$ be a complete, gradient Ricci almost soliton with soliton function $\lambda$. Suppose either $m=2$ or otherwise

$$
\langle\nabla f, \nabla \lambda\rangle \leq 0
$$

Assume the validity of the Poincaré-Sobolev inequality (6.1) for some $0 \leq \alpha<1$ and suppose that for some $p>1$

$$
\int_{B_{r}}|\nabla f|^{p} e^{-f}=o\left(r^{2}\right)
$$

as $r \rightarrow+\infty$. Then either the almost soliton is trivial or

$$
\left\|\lambda_{+}(x)\right\|_{L^{\frac{1}{\alpha}}\left(M, e^{-f}\right)} \geq \frac{4}{S(\alpha)} \frac{\sigma-1-B}{\sigma^{2}} .
$$

Proof. From Corollary 3.2 and (6.2) we deduce

$$
|\nabla f| \Delta_{f}|\nabla f|+\lambda|\nabla f|^{2} \geq 0
$$

Thus we can apply Proposition 6.1 with $p=\sigma, B=0, q(x)=\lambda(x)$ to deduce that either the almost soliton is trivial or (6.3) holds.

Theorem 1.6 now follows immediately from Theorem 6.3. Indeed, since $m \geq 3$, a trivial almost soliton is necessarily Einstein and the soliton function $\lambda$ must be constant. On the other hand, the Poincaré-Sobolev inequality implies that $M$ has infinite volume, therefore $\lambda_{+}=0$. 


\section{Scalar curvature estimates}

In this section we prove Theorem 1.4 stated in the Introduction. Namely, we show that under a pointwise control on the soliton function, the scalar curvature of an almost soliton is bounded from below. Furthermore, the lower bound of the scalar curvature can be estimated both from above and from below and some rigidity at the endpoints occurs.

We recall that a weighted manifold $\left(M,\langle\rangle,, e^{-f} d \mathrm{vol}\right)$ is said to be $f$-parabolic if whenever $w$ is bounded below and satisfies $\Delta_{f} w \leq 0$ then $w$ is constant.

Proof of Theorem 1.4. Since $\mid$ Ric $\left.\right|^{2} \geq S^{2} / m$ by the Cauchy-Schwarz inequality, and $\Delta \lambda \leq 0$ by assumption, (3.15) in Lemma 3.3 yields

$$
\frac{1}{2} \Delta_{f} S=\lambda S-|\mathrm{Ric}|^{2}+(m-1) \Delta \lambda \leq \lambda S-\frac{S^{2}}{m} .
$$

Note next that since $\operatorname{Ric}_{f}=\lambda \geq \lambda_{*}>-\infty$, by Proposition 4.3 we have

$$
\operatorname{vol}_{f}\left(B_{r}\right) \leq C_{1} e^{C_{2} r^{2}},
$$

for some positive constants $C_{1}, C_{2}$, and, in particular,

$$
\liminf _{r \rightarrow \infty} \frac{\log \operatorname{vol}_{f}\left(B_{r}\right)}{r^{2}} \leq C_{2}<+\infty .
$$

Applying [27, Theorem 12] to the function $S_{-}=\max \{-S, 0\}$, which is a weak solution of

$$
\Delta_{f} S_{-} \geq 2 \lambda S_{-}-\frac{2}{m} S_{-}^{2},
$$

with $a(x)=2 \lambda(x), b(x)=\frac{2}{m}, \sigma=2$, and deduce that

$$
S_{-}(x) \leq \sup _{M} \frac{\lambda_{-}(x)}{1 / m},
$$

from which we conclude that

$$
S(x) \geq \min \left\{m \lambda_{*}, 0\right\} .
$$

In particular, $S_{*} \geq 0$ if $\lambda \geq 0$, and $S_{*} \geq m \lambda_{*}$ if $\lambda_{*} \leq \lambda \leq 0$.

Next, again by (7.2), the weak minimum principle at infinity for $\Delta_{f}$ holds (see Theorem 4.4), and therefore we may find a sequence $\left\{x_{n}\right\}$ such that $\Delta_{f} S\left(x_{n}\right) \geq$ $-1 / n$ and $S\left(x_{n}\right) \rightarrow S_{*}$. Computing the liminf of (7.1) along this sequence and setting $\bar{\lambda}=\lim \inf \lambda\left(x_{n}\right)$ we deduce that

$$
0 \leq \bar{\lambda} S_{*}-S_{*}^{2} / m
$$


Thus, if $\bar{\lambda}=0$, then $S_{*}=0$, while if $\bar{\lambda} \neq 0$, then solving the inequality yields $m \bar{\lambda} \leq S_{*} \leq 0$ if $\bar{\lambda}<0$ and $0 \leq S_{*} \leq m \bar{\lambda}$ if $\bar{\lambda}>0$. Since obviously $\lambda_{*} \leq \bar{\lambda} \leq \lambda^{*}$, this gives the scalar curvature estimates in (i), (ii) and (iii).

We now suppose that the scalar curvature achieves its lower bound and, according to the classification in Theorem 2.3, we prove rigidity.

In case (i) using (7.1), we see that $S(x) \geq S_{*}=m \lambda_{*}$ satisfies

$$
\frac{1}{2} \Delta_{f} S \leq-\frac{S}{m}(S-m \lambda) \leq-\frac{S}{m}\left(S-m \lambda_{*}\right)
$$

on the open set $\Omega=\{x \in M: S(x)<0\}$. Therefore, if $S\left(x_{0}\right)=S_{*}=m \lambda_{*}<0$ for some $x_{0}$, we deduce that the function $u=S-m \lambda_{*} \geq 0$ achieves its minimum value $u\left(x_{0}\right)=0$ and satisfies the differential inequality

$$
\frac{1}{2} \Delta_{f} u+\lambda_{*} u \leq 0
$$

on $\Omega$. By the minimum principle, $u(x)=0$ on the connected component $\Omega_{0}$ of $\Omega$ containing $x_{0}$. It follows that the open set $\Omega_{0}$ is also closed, thus $\Omega_{0}=M$ and $u(x)=0$ on $M$. This means that $S(x)=m \lambda_{*}$ is constant. Using this information into (7.3) we get that $\lambda$ is constant. Going back to (7.1), by the equality case in the Cauchy-Schwarz inequality, we obtain

$$
\text { Ric }=\lambda\langle,\rangle,
$$

showing that $M$ is Einstein and the soliton is trivial.

Assume next that the soliton is steady, so that $\lambda \equiv 0$. Then $S(x) \geq S_{*}=0$ solves

$$
\frac{1}{2} \Delta_{f} S \leq 0
$$

Therefore, if $S\left(x_{0}\right)=0$, arguing as above we conclude that $M$ must be Ricci-flat and, by case (a.1) of Theorem 2.3, $M$ is a cylinder over a Ricci-flat, totally geodesic hypersurfaces $\Sigma$.

Finally assume that the almost soliton is shrinking. Then, $S(x) \geq S_{*}=0$ satisfies

$$
\frac{1}{2} \Delta_{f} S \leq \lambda S
$$

If $S\left(x_{0}\right)=0$ for some $x_{0} \in M$, by the minimum principle (see e.g. [10, page 35]) we deduce that $S(x)=0$ is constant and all the inequalities used in (7.1) become equalities. In particular, $|\mathrm{Ric}|^{2}=S^{2} / m=0$ proving that $M$ is Ricci-flat. By case (a.2) in Theorem 2.3, $\lambda$ is a positive constant and $M$ must be isometric to the standard Euclidean space.

It remains to prove the last statement. Suppose then that $S_{*}=m \lambda^{*}>0$. Since $S \geq S_{*}=m \lambda^{*} \geq m \lambda>0$, it follows that $m \lambda S-S^{2} \leq 0$ on $M$. Thus from (7.1),

$$
\Delta_{f} S \leq 0
$$


and $S>0$ is a nonnegative $\Delta_{f}$-superharmonic function. It follows that, if $\left(M,\langle\rangle,, e^{-f} d \mathrm{vol}\right)$ is $f$-parabolic, then $S=m \lambda^{*}$ is constant. Using (7.1) we immediately deduce $S\left(\lambda-\frac{S}{m}\right)=0$ so that $\lambda=\frac{S}{m}$ is constant. From (7.1) we have that $\mid$ Ric $\left.\right|^{2}=\frac{S^{2}}{m}$, and, again by the equality case in the Cauchy-Schwarz inequality

$$
\text { Ric }=\lambda\langle,\rangle,
$$

with $\lambda>0$. Thus $M$ is Einstein and compact by Myers' Theorem. Now from (1.2) and the above considerations it follows that Hess $(f)=0$ on $M$ and compactness implies that $f$ is constant. Finally, if $\lambda \geq A^{2}(1+r)^{-\mu}$ with $0 \leq \mu \leq 1$, then by Proposition $4.3 \operatorname{vol}_{f}\left(\partial B_{r}\right)$ tends to zero as $r \rightarrow \infty$, so, in particular,

$$
\frac{1}{\operatorname{vol}_{f}\left(\partial B_{r}\right)} \notin L^{1}(+\infty)
$$

and adapting to the diffusion operator $\Delta_{f}$ standard proofs valid for the ordinary Laplace-Beltrami operator, [28], one shows that $\left(M,\langle\rangle,, e^{-f} d\right.$ vol), is $f$-parabolic.

\section{A gap theorem for the traceless Ricci tensor}

Proof of Theorem 1.9. As noted in the previous proof, since Ric $f$ is bounded below by $(1.12)$, the weak maximum principle for $\Delta_{f}$ holds on $(M,\langle\rangle,, \nabla f)$. Next, by Corollary 3.6, (1.10), (1.11), and (1.12), we deduce that

$$
\frac{1}{2} \Delta_{f}|T|^{2} \geq 2\left(\lambda_{*}-S^{*} \frac{m-2}{m(m-1)}\right)|T|^{2}-\frac{4}{\sqrt{m(m-1)}}|T|^{3} .
$$

Assuming that $|T|^{*}=\sup _{M}|T|<+\infty$ (for otherwise there is nothing to prove) we may apply the weak maximum principle for $\Delta_{f}$ and deduce that either $|T|^{*}=0$ or $|T|^{*} \geq \frac{1}{2}\left(\sqrt{m(m-1)} \lambda_{*}-S^{*} \frac{m-2}{\sqrt{m(m-1)}}\right)$. In the former case, $T=0$ that is Ric $=S / m\langle$,$\rangle and since m \geq 3, S$ is constant by Schur's lemma and $M$ is Einstein, as required to conclude the proof of Theorem 1.9.

\section{Some topological remarks}

We shall prove the next theorem, which extends results obtained in $[4,5,17,32]$.

Theorem 9.1. Let $\left(M,\langle\rangle,, e^{-f} d \mathrm{vol}\right)$ be a geodesically complete weighted manifold, and assume that there exists a point $o \in M$ and functions $\mu \geq 0$ and $g$ bounded such that for every unit speed geodesic $\gamma$ issuing from $\gamma(0)=\bar{o}$ we have

$$
\operatorname{Ric}_{f}(\dot{\gamma}, \dot{\gamma}) \geq \mu \circ \gamma+\langle\nabla g \circ \gamma, \dot{\gamma}\rangle
$$


and

$$
\int_{0}^{+\infty} \mu \circ \gamma(t) d t=+\infty
$$

Then the following hold:

(a) If the above conditions hold then $\left|\pi_{1}(M)\right|<\infty$.

(b) If in addition $\mathrm{Ric} \leq c<+\infty$ and $\mu=\mu_{o}(r(x))$ is radial, where $r(x)=$ dist $(x, o)$, then $M$ is diffeomorphic to the interior of a compact manifold $N$ with $\partial N \neq \emptyset$.

(c) If $\mu(x) \geq \mu_{0}>0$ and $\sup _{M}(|\nabla f|+|g|) \leq F<+\infty$, then $M$ is compact and $\operatorname{diam}(M) \leq \frac{1}{\mu_{0}}\left[2 F+\sqrt{4 F^{2}+\pi^{2}(m-1) c}\right]$

Clearly the Theorem applies to almost Ricci solitons for which the soliton function $\lambda$ satisfies the conditions listed in the statement.

These three conclusions can be deduced from the following lemmas which estimates the integral of Ric along geodesics.

Lemma 9.2. Let $(M,\langle\rangle$,$) be a Riemannian manifold. Fix o \in M$ and let $r(x)=$ $\operatorname{dist}(x, o)$. For any point $q \in M$, let $\gamma_{q}:[0, r(q)] \rightarrow M$ be a minimizing geodesic from o to $q$ such that $\left|\dot{\gamma}_{q}\right|=1$.

(A) If $h \in \operatorname{Lip}_{\text {loc }}(\mathbb{R})$ is such that $h \geq 0$ and $h(0)=0$, then, for every $q \notin \operatorname{cut}(o)$,

$$
h^{2}(r(q)) \Delta r(q) \leq(m-1) \int_{0}^{r(q)}\left(h^{\prime}\right)^{2} d s-\int_{0}^{r(q)} h^{2} \operatorname{Ric}\left(\dot{\gamma}_{q}, \dot{\gamma}_{q}\right) d s .
$$

If in addition $h(r(q))=0$, then for every $q \in M$,

$$
0 \leq(m-1) \int_{0}^{r(q)}\left(h^{\prime}\right)^{2} d s-\int_{0}^{r(q)} h^{2} \operatorname{Ric}\left(\dot{\gamma}_{q}, \dot{\gamma}_{q}\right) d s
$$

(B) For all $q \in M$ such that $r(q)>2$, we have

$$
\int_{0}^{r(q)} \operatorname{Ric}\left(\dot{\gamma}_{q}, \dot{\gamma}_{q}\right) \leq 2(m-1)+H_{o}+H_{q}
$$

where, as in [32], we have set

$$
H_{p}=\max \left\{0, \sup _{B_{1}(p)} \operatorname{Ric}\right\}, \forall p \in M
$$

Proof. Part (A) is well known. We provide a proof which avoids the use of the second variation formula for arc-length. 
Fix a point $q \in M$ and suppose that $q \notin \operatorname{cut}(o)$. Proceeding as in the proof of Theorem 4.1 we rewrite (4.7) as

$$
\frac{\left(\Delta r \circ \gamma_{q}\right)^{2}}{m-1}+\frac{d}{d s}\left(\Delta r \circ \gamma_{q}\right)+\operatorname{Ric}\left(\dot{\gamma}_{q}, \dot{\gamma}_{q}\right) \leq 0 .
$$

If $h \in \operatorname{Lip}_{\text {loc }}(\mathbb{R})$ is such that $h \geq 0, h(0)=0$, multiplying by $h^{2}$ equation (9.1) and integrating on $[0, t]$ we get

$$
\int_{0}^{t} h^{2} \frac{\left(\Delta r \circ \gamma_{q}\right)^{2}}{m-1} d s+\int_{0}^{t} \frac{d}{d s}\left(\Delta r \circ \gamma_{q}\right) h^{2} d s+\int_{0}^{t} h^{2} \operatorname{Ric}\left(\dot{\gamma}_{q}, \dot{\gamma}_{q}\right) d s \leq 0 .
$$

Integrating by parts and noting that $\left(\Delta r \circ \gamma_{q}\right) h^{2} \rightarrow 0$ as $r \rightarrow 0$ we obtain

$$
\begin{aligned}
0 \geq & \int_{0}^{t} h^{2} \frac{\left(\Delta r \circ \gamma_{q}\right)^{2}}{m-1} d s+h^{2}(t) \Delta r \circ \gamma_{q}(t) \\
& -2 \int_{0}^{t} h h^{\prime}\left(\Delta r \circ \gamma_{q}\right) d s+\int_{0}^{t} \operatorname{Ric}\left(\dot{\gamma_{q}}, \dot{\gamma_{q}}\right) h^{2} d s .
\end{aligned}
$$

Since

$$
\begin{aligned}
2 h h^{\prime}\left(\Delta r \circ \gamma_{q}\right) & =\frac{2 h \Delta r \circ \gamma_{q}}{\sqrt{m-1}} \sqrt{m-1} h^{\prime} \\
& \leq \frac{h^{2}\left(\Delta r \circ \gamma_{q}\right)^{2}}{m-1}+(m-1)\left(h^{\prime}\right)^{2},
\end{aligned}
$$

we deduce that

$$
0 \geq h^{2}(t) \Delta r \circ \gamma_{q}(t)-(m-1) \int_{0}^{t}\left(h^{\prime}\right)^{2} d s+\int_{0}^{t} h^{2} \operatorname{Ric}\left(\dot{\gamma_{q}}, \dot{\gamma_{q}}\right) d s
$$

and, setting $t=r(q)$, we conclude that

$$
h^{2}(r(q)) \Delta r(q) \leq(m-1) \int_{0}^{r(q)}\left(h^{\prime}\right)^{2} d s-\int_{0}^{r(q)} h^{2} \operatorname{Ric}\left(\dot{\gamma_{q}}, \dot{\gamma_{q}}\right) d s .
$$

If in addition $h$ satisfies $h^{2}(r(q))=0$, then the above inequality becomes

$$
0 \leq(m-1) \int_{0}^{r(q)}\left(h^{\prime}\right)^{2} d s-\int_{0}^{r(q)} h^{2} \operatorname{Ric}\left(\dot{\gamma_{q}}, \dot{\gamma_{q}}\right) d s
$$

This completes the proof if $q \notin$ cut $(o)$. In the general case inequality (9.2) can be extended to any $q \in M$ using the Calabi trick. Indeed, suppose that $q \in \operatorname{cut}(o)$. Translating the origin $o$ to $o_{\varepsilon}=\gamma_{q}(\varepsilon)$ so that $q \notin$ cut $\left(o_{\varepsilon}\right)$, using the triangle 
inequality and, finally, taking the limit as $\varepsilon \rightarrow 0$, one checks that (9.2) holds also in this case.

To prove part (B) we note that if $h \in \operatorname{Lip}_{\text {loc }}(\mathbb{R})$ is such that $h \geq 0$ and $h(0)=$ $h(r(q))=0$, then by we may rewrite (A) in the form

$$
\int_{0}^{r(q)} \operatorname{Ric}\left(\dot{\gamma}_{q}, \dot{\gamma}_{q}\right) d s \leq(m-1) \int_{0}^{r(q)}\left(h^{\prime}\right)^{2} d s+\int_{0}^{r(q)}\left(1-h^{2}\right) \operatorname{Ric}\left(\dot{\gamma}_{q}, \dot{\gamma}_{q}\right) d s .
$$

Choosing

$$
h(s)= \begin{cases}s & 0 \leq s \leq 1 \\ 1 & 1 \leq s \leq r(q)-1 \\ r(q)-s & r(q)-1 \leq s \leq r(q)\end{cases}
$$

where $r(q)>2$, we obtain

$$
\begin{aligned}
\int_{0}^{r(q)} \operatorname{Ric}\left(\dot{\gamma}_{q}, \dot{\gamma}_{q}\right) d s \leq & 2(m-1)+\int_{0}^{1}\left(1-s^{2}\right) \operatorname{Ric}\left(\dot{\gamma}_{q}, \dot{\gamma}_{q}\right) d s \\
& +\int_{r(q)-1}^{r(q)}\left(1-(r(q)-s)^{2}\right) \operatorname{Ric}\left(\dot{\gamma}_{q}, \dot{\gamma}_{q}\right) d s \\
\leq & 2(m-1)+H_{o}+H_{q} .
\end{aligned}
$$

Lemma 9.3. Let $\left(M,\langle\rangle,, e^{-f} d \mathrm{vol}\right)$ be a complete weighted Riemannian manifold. Fix $o \in M$ and let $r(x)=\operatorname{dist}(x, o)$ and assume that there exist functions $\mu$ and $g$ bounded such that for every unit speed geodesic $\gamma$ issuing from $o$

$$
\operatorname{Ric}_{f}(\dot{\gamma}, \dot{\gamma}) \geq \mu(\gamma(t))+\langle\nabla g, \dot{\gamma}\rangle \text {. }
$$

Then for every such geodesic

$$
\begin{aligned}
\int_{0}^{t} \operatorname{Ric}(\dot{\gamma}, \dot{\gamma})= & \langle\nabla f, \dot{\gamma}(0)\rangle-\langle\nabla f, \dot{\gamma}(t)\rangle+\int_{0}^{t} \mu(\gamma(s)) d s+g(\gamma(t))-g(o) \\
& \geq-\left|\nabla f_{\gamma(0)}\right|-\left|\nabla f_{\gamma(t)}\right|-2 \sup |g|+\int_{0}^{t} \mu(\gamma(s)) d s .
\end{aligned}
$$

Proof. By assumption

$$
\operatorname{Ric}(\dot{\gamma}, \dot{\gamma})+\operatorname{Hess}(f)(\dot{\gamma}, \dot{\gamma}) \geq \mu \circ \gamma+\langle\nabla g, \dot{\gamma}\rangle,
$$

which can be written in the form

$$
\operatorname{Ric}(\dot{\gamma}, \dot{\gamma})+\frac{d}{d t}\langle\nabla f(\gamma), \dot{\gamma}\rangle \geq \mu \circ \gamma+\frac{d}{d t}(g \circ \gamma) \text {. }
$$

Now integrating on $[0, t]$,

$$
\int_{0}^{t} \operatorname{Ric}(\dot{\gamma}, \dot{\gamma})+\langle\nabla f, \dot{\gamma}(t)\rangle-\langle\nabla f, \dot{\gamma}(0)\rangle \geq \int_{0}^{t} \mu(\gamma(s)) d s+g(\gamma(t))-g(o) \text {. }
$$


We are now in the position to give the:

Proof of Theorem 9.1. Following [32], let us consider the Riemannian universal covering $P: M^{\prime} \rightarrow M$ of $M$. Since $P$ is a local isometry then $M^{\prime}$ is a weighted complete Riemannian manifold with weight $f^{\prime}=f \circ P$. Moreover, since every unit speed geodesic $\gamma^{\prime}$ projects to a unit speed geodesic $\gamma=P \circ \gamma^{\prime}$ we see that

$$
\operatorname{Ric}_{f^{\prime}}^{\prime}\left(\dot{\gamma}^{\prime}, \dot{\gamma}^{\prime}\right)=\operatorname{Ric}_{f}(\dot{\gamma}, \dot{\gamma}) \geq \mu \circ \gamma+\frac{d}{d t}(g \circ \gamma)=\mu^{\prime} \circ \gamma^{\prime}+\frac{d}{d t}\left(g^{\prime} \circ \gamma^{\prime}\right),
$$

where the function $g^{\prime}=g \circ P$ is bounded and $\mu^{\prime}=\mu \circ P \geq 0$ satisfies

$$
\int_{0}^{+\infty} \mu^{\prime} \circ \gamma^{\prime}(t) d t=\int_{0}^{+\infty} \mu \circ \gamma(t) d t=+\infty .
$$

We identify

$$
\pi_{1}(M, o)=\operatorname{Deck}\left(M^{\prime}\right),
$$

the covering transformation group, and recall that there is a bijective correspondence $\pi_{1}(M, o) \longleftrightarrow P^{-1}(o)$. Therefore it suffices to show that $P^{-1}(o) \subset$ $B_{R}^{\prime}\left(o^{\prime}\right)$ for some $R \gg 1$. Since $\pi_{1}(M, o)=\operatorname{Deck}\left(M^{\prime}\right)$ acts transitively on the fibre $P^{-1}(o)$, we have

$$
P^{-1}(o)=\left\{h\left(o^{\prime}\right): h \in \operatorname{Deck}\left(M^{\prime}\right)\right\},
$$

and we are reduced to showing that

$$
r^{\prime}\left(h\left(o^{\prime}\right)\right) \leq R<\infty, \quad \forall h \in \operatorname{Deck}\left(M^{\prime}\right),
$$

where we have set $r^{\prime}\left(x^{\prime}\right)=\operatorname{dist}_{M^{\prime}}\left(o^{\prime}, x^{\prime}\right)$. Fix $h \in \operatorname{Deck}\left(M^{\prime}\right)$ and a unit speed, minimizing geodesic $\gamma_{h\left(o^{\prime}\right)}^{\prime}:\left[0, r^{\prime}\left(h\left(o^{\prime}\right)\right)\right] \rightarrow M^{\prime}$, issuing from $\gamma_{h\left(o^{\prime}\right)}^{\prime}(0)=o^{\prime}$. Recalling that $\operatorname{Ric}^{\prime}\left(\dot{\gamma}^{\prime}, \dot{\gamma}^{\prime}\right)=\operatorname{Ric}_{f^{\prime}}^{\prime}\left(\dot{\gamma}^{\prime}, \dot{\gamma}^{\prime}\right)-\frac{d}{d t}\left\langle\nabla^{\prime} f^{\prime} \circ \gamma^{\prime}, \dot{\gamma}^{\prime}\right\rangle$ and using Lemma 9.2 (B) and Lemma 9.3 we get

$$
\begin{aligned}
\int_{0}^{r^{\prime}\left(h\left(o^{\prime}\right)\right)} \mu^{\prime} \circ \gamma_{h\left(o^{\prime}\right)}^{\prime}(s) d s \leq & 2(m-1)+H_{o^{\prime}}+H_{h\left(o^{\prime}\right)} \\
& +\left|\nabla^{\prime} f^{\prime}\right|\left(o^{\prime}\right)+\left|\nabla^{\prime} f^{\prime}\right|\left(h\left(o^{\prime}\right)\right)+2 \sup _{M^{\prime}}\left|g^{\prime}\right| .
\end{aligned}
$$

Since $P: M^{\prime} \rightarrow M$ is a local isometry and $o^{\prime}, h\left(o^{\prime}\right) \in P^{-1}(o)$ we deduce

$$
\left|\nabla^{\prime} f^{\prime}\right|\left(o^{\prime}\right)=|\nabla f|(o)=\left|\nabla^{\prime} f^{\prime}\right|\left(h\left(o^{\prime}\right)\right) .
$$

On the other hand $\operatorname{Deck}\left(M^{\prime}\right) \subset \operatorname{Iso}\left(M^{\prime}\right)$, so $h\left(B_{1}^{\prime}\left(o^{\prime}\right)\right)$ is isometric to $B_{1}^{\prime}\left(h\left(o^{\prime}\right)\right)$ and we have

$$
\left|H_{o^{\prime}}\right|=\left|H_{h\left(o^{\prime}\right)}\right| \text {. }
$$


Summarizing, we have obtained that, for every $h \in \operatorname{Deck}\left(M^{\prime}\right)$,

$$
\int_{0}^{r^{\prime}\left(h\left(o^{\prime}\right)\right)} \mu^{\prime} \circ \gamma_{h\left(o^{\prime}\right)}^{\prime}(s) d s \leq 2\left\{(m-1)+H_{o^{\prime}}+|\nabla f|(o)\right\}+2 \sup _{M}|g| .
$$

With this preparation, we now argue by contradiction and suppose that there exists a sequence of transformations $\left\{h_{n}\right\} \subset \operatorname{Deck}\left(M^{\prime}\right)$ such that

$$
r^{\prime}\left(h_{n}\left(o^{\prime}\right)\right) \rightarrow+\infty \text {, as } n \rightarrow+\infty \text {. }
$$

Let $\gamma_{h_{n}\left(o^{\prime}\right)}^{\prime}(s)=\exp _{o^{\prime}}\left(s \xi_{n}^{\prime}\right)$, where $\left\{\xi_{n}^{\prime}\right\} \subset \mathbb{S}_{o^{\prime}}^{m-1} \subset T_{o^{\prime}} M^{\prime}$. Then, there exists a subsequence $\left\{\xi_{n_{k}}^{\prime}\right\} \rightarrow \xi^{\prime} \in \mathbb{S}_{o^{\prime}}^{m-1}$ as $k \rightarrow+\infty$ and, by the Ascoli-Arzelà's Theorem, the sequence of minimizing geodesics $\left\{\gamma_{h_{n_{k}}\left(o^{\prime}\right)}^{\prime}\right\}$ converges uniformly on compact subintervals of $[0,+\infty)$ to the unit speed geodesic $\gamma^{\prime}(s)=\exp _{o^{\prime}}\left(s \xi^{\prime}\right)$. Since, by (9.4)

$$
\int_{0}^{+\infty} \mu^{\prime} \circ \gamma^{\prime}(s) d s=+\infty
$$

we can choose $T \gg 1$ such that

$$
\int_{0}^{T} \mu^{\prime} \circ \gamma^{\prime}(s) d s>2\left\{(m-1)+H_{o^{\prime}}+|\nabla f|(o)\right\}+\underset{M}{2 \sup _{M}|g| .}
$$

On the other hand, according to (9.6) we find $k_{0}>0$ such that, for every $k \geq k_{0}$, $r^{\prime}\left(h_{n_{k}}\left(o^{\prime}\right)\right)>T$. It follows from this, from inequality (9.5) and the definition of $\mu^{\prime}\left(x^{\prime}\right)=\mu \circ P\left(x^{\prime}\right) \geq 0$ that

$$
\begin{aligned}
\int_{0}^{T} \mu^{\prime} \circ \gamma_{h_{n_{k}}\left(o^{\prime}\right)}^{\prime}(s) d s & \leq \int_{0}^{r^{\prime}\left(h_{n_{k}}\left(o^{\prime}\right)\right)} \mu^{\prime} \circ \gamma_{h_{n_{k}}\left(o^{\prime}\right)}^{\prime}(s) d s \\
& \leq 2\left\{(m-1)+H_{o^{\prime}}+|\nabla f|(o)\right\}+2 \sup _{M}|g| .
\end{aligned}
$$

Whence, letting $k \rightarrow+\infty$ we deduce

$$
\int_{0}^{T} \mu^{\prime} \circ \gamma^{\prime}(s) d s \leq 2\left\{(m-1)+H_{o^{\prime}}+|\nabla f|(o)\right\}+\underset{M}{2 \sup _{M}|g|}
$$

which contradicts (9.7).

Now for the proof of (b), suppose Ric $\leq c$. Fix $q \in M$ such that $r(q)=$ $\operatorname{dist}(o, q)>2$, and let $\gamma_{q}$ be a minimizing geodesic joining $o$ to $q$. As above, combining (B) of Lemma 9.2 and Lemma 9.3, and recalling that $\mu(x)=\mu_{o}(r(x))$ is radial we obtain

$$
\begin{aligned}
-|\nabla f(o)|-|\nabla f(\gamma(r(q)))|-2 \sup _{M}|g|+\int_{0}^{r(q)} \mu_{o}(s) d s & \leq 2(m-1)+H_{q}+H_{o} \\
& \leq 2(m-1)+2 c,
\end{aligned}
$$


which implies

$$
|\nabla f(q)| \geq \int_{0}^{r(q)} \mu_{o}(s) d s+\{-|\nabla f(o)|-2(m-1)-2 c\}-2 \sup _{M}|g| .
$$

Since $0<\mu_{o} \notin L^{1}(+\infty)$ if $r(q) \gg 1$, say $r(q) \geq R_{0}$, we have $|\nabla f(q)|>0$. Thus $f$ has no critical point in $M \backslash B_{R_{0}}(o)$. Again from Lemmas 9.2 and 9.3, for every $0 \leq t \leq r(q)$,

$$
\int_{0}^{t} \mu_{o}(s) d s-\left\langle\nabla f \circ \gamma_{q}, \dot{\gamma}_{q}\right\rangle+\left\langle\nabla f \circ \gamma_{q}, \dot{\gamma}_{q}\right\rangle_{\left.\right|_{s=0}}+g(q)-g(o) \leq 2(m-1)+2 c,
$$

so that

$$
\left.\frac{d}{d s} f \circ \gamma_{q}\right|_{s=t} \geq \int_{0}^{t} \mu_{o}(s) d s-\left\{|\nabla f(o)|+2 \sup _{M}|g|+2(m-1)+2 c\right\} .
$$

Thus, integrating on $[2, r(q)]$,

$$
\begin{aligned}
& f(q) \geq \int_{2}^{r(q)} \int_{0}^{t} \mu_{o}(s) d s-\left|f\left(\gamma_{q}(2)\right)\right| \\
& -\left\{|\nabla f(o)|+2 \sup _{M}|g|+2(m-1) 2 c\right\}(r(q)-2) \\
& \geq \int_{2}^{r(q)} \int_{0}^{r(q)} \mu_{o}(s) d s-\max _{\partial B_{2}(o)}|f| \\
& -\left\{|\nabla f(o)|+2 \sup _{M}|g|+2(m-1)+2 c\right\}(r(q)-2) \rightarrow+\infty \text {, }
\end{aligned}
$$

for $r(q) \rightarrow+\infty$. Therefore $f$ is a smooth exhaustion function whose critical points are confined in a compact set. By standard Morse theory, there exists a compact manifold $N$ with boundary such that $M$ is diffeomorphic to the interior of $N$.

Finally, we prove (c). Suppose that $\sup _{M}(|\nabla f|+|g|) \leq F<+\infty$. Then, by (9.3) in Lemma 9.3, for every unit speed geodesic $\gamma$ issuing from $o$ we have

$$
\operatorname{Ric}(\dot{\gamma}, \dot{\gamma}) \geq \mu_{0}+\frac{d}{d t} G \circ \gamma
$$

where $G \circ \gamma=-\langle\nabla f \circ \gamma, \dot{\gamma}\rangle+g \circ \gamma$ satisfies $|G \circ \gamma| \leq \sup _{M}(|\nabla f|+|g|)$. Using Theorem 1.2 in [8] we obtain the desired diameter estimate. 


\section{References}

[1] J. CASE, Y.-J. ShU and G. WeI, Rigidity of quasi-Einstein metrics, Differential Geom. Appl. 29 (2011), 93-100.

[2] B. L. Chen, Strong uniqueness of the Ricci flow, J. Differential Geom. 82 (2009), 362-382.

[3] H. D. CAO, B. L. CHEN and X. P. ZHU, "Recent Developments on Hamilton's Ricci flow", Surveys in Differential Geometry XII, 2008.

[4] M. Eminenti, G. La Nave and C. Mantegazza, Ricci Solitons: the equation point of view, Manuscripta Math. 127 (2008), 345-367.

[5] M. FERnÁndeZ-LóPEZ and E. GARCía-Río, A remark on compact Ricci solitons, Math. Ann. 340 (2008), 893-896.

[6] M. Fernández-López and E. García Río, Maximum principles and gradient Ricci solitons, J. Differential Equations 251 (2011), 73-81.

[7] M. FERnÁNDEZ-LóPEZ and E. GARCÍA-RÍo, Rigidity of shrinking Ricci solitons, Math. Z. 269 (2011), 461-466.

[8] G. J. Galloway, A generalization of Myers' theorem and an application to relativistic cosmology, J. Differential Geom. 14 (1979), 105-116.

[9] R. GReENE and H. H. Wu, "Function Theory on Manifolds which posses a pole", Lect. Notes in Math., Vol. 699, Springer-Verlag, Berlin, 1979.

[10] D. Gilbarg and N. Trudinger, "Elliptic Partial Differential Equations of Second Order", second edition, Springer-Verlag, Berlin, 1983.

[11] R. S. Hamilton, The Ricci flow on surfaces, In: "Mathematics and general relativity" (Santa Cruz, CA, 1986), Contemp. Math., Vol. 71. American Mathematical Society, Providence, pp. 237-262 (1988)

[12] S. ISHIHARA and Y. TASHIRO, On Riemannian manifolds admitting a concircular transformation, Math. J. Okayama Univ. 9 (1959/1960), 19-47.

[13] M. KANAI, On a differential equation characterizing a Riemannian structure of a manifold, Tokyo J. Math. 6 (1983), 143-151.

[14] L. MARI, M. Rigoli and A. G. SetTI, Keller-Osserman conditions for diffusion-type operators on Riemannian manifolds, J. Funct. Anal. 258 (2010), 665-712.

[15] G. Maschler, Special Kähler-Ricci potentials and Ricci solitons, Ann. Global Anal. Geom. 34 (2008), 367-380.

[16] O. Munteanu and N. Sesum, On gradient Ricci solitons, J. Geom. Anal. DOI: $10.1007 / \mathrm{s} 12220-011-9252-6$.

[17] A. NABER, Noncompact shrinking 4-solitons with nonnegative curvature, J. Reine Angew. Math. 645 (2010), 125-153.

[18] M. OBATA, Certain conditions for a Riemannian manifold to be isometric with a sphere, J. Math. Soc. Japan 14 (1962), 333-340.

[19] M. OKUMURA, Hypersurfaces and a pinching problem on the second fundamental tensor, Amer. J. Math 96 (1974), 207-213.

[20] B. O'NeILl, "Semi-Riemannian Geometry with Applications to Relativity", Pure and Applied Mathematics, Vol. 103, Academic Press, San Diego, 1983.

[21] G. Perelman, Ricci flow with surgery on three manifolds, arXiv: math.DG/0303109.

[22] P. PETERSEN and W. WyLIE, On the classification of gradient Ricci solitons, Geom. Topol. 14 (2010), 2277-2300.

[23] P. Petersen and W. Wylie, Rigidity of gradient Ricci solitons, Pacific J. Math. 241 (2009), 329-345.

[24] S. Pigola, M. Rigoli and A. G. Setti, A remark on stochastic completeness and the maximum principle, Proc. Amer. Math. Soc.131 (2003), 1283-1288.

[25] S. Pigola, M. Rigoli and A. G. SetTi, "Maximum Principles on Riemannian Manifolds and Applications", Mem. Amer. Math Soc., Vol. 174, 2005, no. 822, x+99 pp. 
[26] S. Pigola, M. Rigoli and A. G. Setti, "Vanishing and Finiteness Results in Geometric Analysis: a Generalization of the Bochner technique", Progress in Mathematics, Vol. 266, 2008, Birkhäuser.

[27] S. Pigola, M. Rimoldi and A. G. SETti, Remarks on non-compact gradient Ricci solitons, Math. Z. 258 (2011), 347-362.

[28] M. Rigoli and A. G. SETti, Liouville type theorems for $\varphi$-subharmonic functions, Rev. Mat. Iberoamericana 17 (2001), 471-520.

[29] A. G. SETTI, Eigenvalue estimates for the weighted Laplacian on a Riemannian manifold, Rend. Sem. Mat. Univ. Padova 100 (1998), 27-55.

[30] Y. TASHIRO, Complete Riemannian manifolds and some vector fields, Trans. Amer. Math. Soc. 117 (1965), 251-275.

[31] G. WEI and W. WYLIE, Comparison geometry for the Bakry-Emery Ricci tensor, J. Differential Geom. 83 (2009), 377-405.

[32] W. WYLIE, Complete shrinking Ricci solitons have finite fundamental group, Proc. Amer. Math Soc. 136 (2008), no. 5, 1803-1806

[33] Z.-H. ZHANG, Gradient shrinking solitons with vanishing Weyl tensor, Pacific J. Math. 242 (2009), 189-200.

[34] Z.-H. ZHANG, On the completeness of gradient Ricci solitons, Proc. Amer. Math. Soc. 137 (2009), 2755-2759.

[35] S. ZHANG, On a sharp volume estimate for gradient Ricci solitons with scalar curvature bounded below, Acta Math. Sin. (Engl. Ser.) 27 (2011), 871-882.

Sezione di Matematica - DiSAT

Università dell'Insubria - Como

Via Valleggio, 11

I-22100 Como, Italia

stefano.pigola@uninsubria.it

Dipartimento di Matematica

Università degli Studi di Milano

Via Saldini, 50

I-20133 Milano, Italia

marco.rigoli@unimi.it

Dipartimento di Matematica

Università degli Studi di Milano

Via Saldini, 50

I-20133 Milano, Italia

michele.rimoldi@unimi.it

Sezione di Matematica - DiSAT

Università dell'Insubria - Como

Via Valleggio, 11

I-22100 Como, Italia

alberto.setti@uninsubria.it 